This item was submitted to Loughborough's Research Repository by the author.

Items in Figshare are protected by copyright, with all rights reserved, unless otherwise indicated.

\title{
Wave-power absorption from a finite array of oscillating wave surge converters
}

PLEASE CITE THE PUBLISHED VERSION

http://dx.doi.org/10.1016/j.renene.2013.08.046

PUBLISHER

(C) Elsevier

VERSION

AM (Accepted Manuscript)

\section{PUBLISHER STATEMENT}

This work is made available according to the conditions of the Creative Commons Attribution-NonCommercialNoDerivatives 4.0 International (CC BY-NC-ND 4.0) licence. Full details of this licence are available at: https://creativecommons.org/licenses/by-nc-nd/4.0/

\section{LICENCE}

CC BY-NC-ND 4.0

\section{REPOSITORY RECORD}

Renzi, Emiliano, A. Abdolali, G. Bellotti, and F. Dias. 2019. "Wave-power Absorption from a Finite Array of Oscillating Wave Surge Converters". figshare. https://hdl.handle.net/2134/17132. 


\title{
Wave-power absorption from a finite array of oscillating wave surge converters
}

\author{
E. Renzii ${ }^{\mathrm{a}, *}$, A. Abdolali ${ }^{\mathrm{b}}$, G. Bellotti ${ }^{\mathrm{b}}$, F. Dias ${ }^{\mathrm{a}, \mathrm{c}}$ \\ ${ }^{a}$ UCD School of Mathematical Sciences, University College Dublin, Belfield Dublin 4, \\ Ireland \\ ${ }^{b}$ Dipartimento di Scienze dell'Ingegneria Civile (DSIC), Università di Roma Tre, via \\ Vito Volterra 62, 00146, Rome, Italy \\ ${ }^{c}$ Centre de Mathématiques et de Leurs Applications (CMLA), Ecole Normale Supérieure \\ de Cachan, 94235 Cachan, France
}

\begin{abstract}
Semi-analytical and fully numerical modelling is developed in the framework of the inviscid potential flow theory to investigate the dynamics of a wave farm made by flap-type wave energy converters in the nearshore. The hydrodynamic parameters and the efficiency of the system in typical layouts are calculated with both models. Good agreement is shown between the two approaches. Parametric analysis undertaken with the semi-analytical model allows to identify a near-resonant phenomenon which is responsible for increasing the absorbed power by the single elements of the array. Such result could be used as a preliminary design criterion. The numerical model is then applied to analyse a configuration of practical engineering interest, i.e. an array of two staggered converters. The dynamics arising in this more complex system is explained, showing that non-symmetric layouts can be less effective.
\end{abstract}

Keywords: Wave energy, wave-structure interaction, arrays, Oscillating Wave Surge Converter, numerical modelling

\footnotetext{
* Corresponding author

Email addresses: emiliano.renzi@ucd.ie (E. Renzi), ali.abdolali@uniroma3.it (A. Abdolali), giorgio.bellotti@uniroma3.it (G. Bellotti), frederic.dias@ucd.ie (F. Dias)
} 


\section{Introduction}

Commercialisation of wave energy systems requires the deployment of wave energy converters (WECs) in large arrays as a fundamental market acceleration strategy. When working together in an array, WECs can interfere in either a constructive or destructive way, depending on the geometric layout and their mutual distance (see [1]-[10]). In this paper we investigate the dynamic interactions arising within an array of large flap-type WECs, namely the Oscillating Wave Surge Converters (OWSCs). Each OWSC is made by a flap hinged on a foundation at the bottom of the ocean and pitching under the action of incident waves in the nearshore [11]. Examples of OWSC at an advanced stage of design are the WaveRoller ${ }^{\mathrm{TM}}$ developed by AW Energy (http://aw-energy.com) and the Oyster $800^{\mathrm{TM}}$ WEC developed by Aquamarine Power Limited (APL, www.aquamarinepower.com). In order to investigate the behaviour of an array of several OWSCs, four quantities are essential: the characteristic wave amplitude and wavenumber, $A$ and $k$ respectively, the characteristic width of the converters $b$ and their mutual characteristic distance $s$. Various parameters can be formed from those quantities which are used to identify the regime of the system: $A / b, k b, k s$. First, in this paper we shall restrict our analysis to monochromatic waves of smallamplitude, for which $A / b \ll 1$. Second, we shall consider large flaps, so that $k b=O(1)$, and intermediate spacing between them, for which $k s=O(1)$. With the assumption $A / b \ll 1$, the behaviour of the system can be described by recurring to the linearised versions of the inviscid-irrotational equations of motion (potential-flow model, see for example [12]). Such hypotheses do not allow to consider either random-sea, vortex-shedding and nonlinear diffraction effects, which are currently being investigated with the aid of different models $[13,14,15,16,17]$. Yet the linearised potential-flow model provides an insightful description of the system dynamics which is fundamental for the successful design of such a costly project. Another important parameter to characterise the system regime is the product $k b$ between the wavenumber of the incident wave and the characteristic width of the converters. Several existing analytical models are indeed applicable to the OWSC in the limiting cases $k b \ll 1$ and $k b \gg 1$. The first case corresponds to the socalled "point-absorber" approximation $[1,3]$, while the second one refers to the "line-absorber" limit [9]. However, considering an average OWSC width $b \simeq 30 \mathrm{~m}$ and a characteristic wavelength $\lambda=2 \pi / k \simeq 100 \mathrm{~m}$ (see for example [11]), yields $k b=O(1)$, which falls outside the limits of applicability of 
the aforementioned theories. Recently, new models in the regime $k b=O(1)$ have been developed to investigate the behaviour of an OWSC in a channel $[18,19]$, an infinite array of OWSCs [20] and a single OWSC in the open ocean $[21,22,23]$. However, the analysis of a finite array of OWSCs seems not to have been undertaken yet. Indeed several theoretical models are available to investigate the interactions in an array of floating bodies which are also implemented in numerical routines (see for example [5] and [24]-[30]). Some of these models rely on simplifying assumptions on the parameter $k s$. For $k s \ll 1$, the spacing between the elements can be neglected without appreciable consequences, as shown by Mei et al. [25] and Adamo \& Mei [29] for an array of closely-spaced storm gates. On the other hand, when $k s \gg 1$ the wide-spacing approximation can be applied, for which radially outgoing waves are approximated as plane waves [5]. Here we shall consider the intermediate case $k s=O(1)$, where interference effects between the elements of the array must be appropriately accounted for [12].

In this paper, a twofold analytical and numerical approach is undertaken to investigate the dynamics of a finite array of OWSCs in the open ocean. In $\S 2$ a general mathematical model of the system is introduced and the governing equations are detailed. Then a new semi-analytical model for an in-line array of OWSCs in normally incident waves is derived. This model, yet necessarily based on some simplifying assumptions, provides a valuable physical insight on the system dynamics. In addition to the analytical approach, a finite-element model is presented to solve numerically the governing equations of the system for more general layouts. Then in $\S 3$ both models are validated. The dynamics of two and three in-line converters is discussed. For the first time, a near-resonant phenomenon - already known for arrays of floats - is shown to occur for an array of OWSCs. Hence the semi-analytical solution is employed in $\S 3.2$ to investigate the parametric behaviour of the system with respect to the period of the incident wave and the spacing between the flaps, showing potential for constructive interaction at near-resonant periods. Finally, in $\S 4$ the potential of the numerical model is applied to analyse a configuration of practical engineering interest, i.e. an array of two staggered OWSCs. 

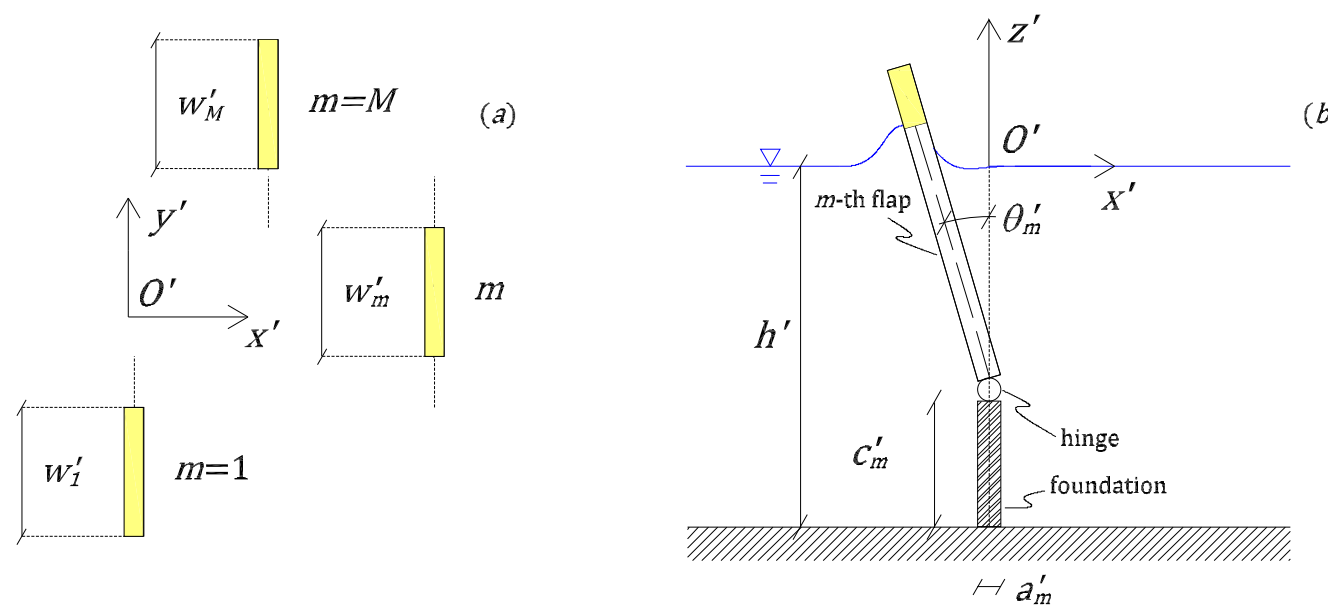

Figure 1: Geometry of an array made by $M$ OWSCs. (a) plan view, (b) section of the $m$-th OWSC.

\section{Mathematical model}

\subsection{Governing equations}

Referring to figure 1, consider a system made by a finite number $M$ of OWSCs in an ocean of finite depth $h^{\prime}$. Let the primes denote physical quantities. Each OWSC is modelled as a rectangular flap of thickness $a_{m}^{\prime}$ and width $w_{m}^{\prime}$, hinged on a bottom foundation of height $c_{m}^{\prime}, m=1,2, \ldots, M$. 77 Under the action of monochromatic incident waves of amplitude $A_{I}^{\prime}$ and 78 period $T^{\prime}$, the flaps are able to perform a pitching motion, from which useful 9 energy is extracted by means of linear power take-off (PTO) mechanisms o linked to each device. Given the linearity of the system, all flaps oscillate 1 with the same period $T^{\prime}$ but with potentially different phases, depending ${ }_{2}$ on the geometry. A Cartesian system of reference $O^{\prime}\left(x^{\prime}, y^{\prime}, z^{\prime}\right)$ is set at an 3 arbitrary origin $O^{\prime}$ on the still water level $z^{\prime}=0$, with the centreline of each flap aligned with the $y^{\prime}$ axis and the $z^{\prime}$ axis pointing vertically upwards (see ${ }_{5}$ again figure 1). Assume that the $m$ th flap is able to perform oscillations of 6 angular amplitude $\theta_{m}^{\prime}\left(t^{\prime}\right)$ about the $y^{\prime}$ axis at $z^{\prime}=-h^{\prime}+c_{m}^{\prime} ; t^{\prime}$ is time. Now let ${ }^{7} \mathcal{L}_{m}^{\prime}$ denote the region occupied by the $m$ th flap at the equilibrium position s (i.e. $\theta_{m}^{\prime}=0$ ) and let $\partial \mathcal{L}_{m}^{\prime}$ be its solid boundary. Assume that the fluid 9 is inviscid and incompressible and the flow irrotational. Then there exists a potential $\Phi^{\prime}$ for the velocity field $\mathbf{v}^{\prime}=\nabla^{\prime} \Phi^{\prime}$ which satisfies the Laplace 1 equation

$$
\nabla^{\prime 2} \Phi^{\prime}\left(x^{\prime}, y^{\prime}, z^{\prime}, t^{\prime}\right)=0
$$


in the fluid domain. In (1), $\nabla^{\prime} f^{\prime}=\left(f_{, x^{\prime}}^{\prime}, f_{y^{\prime}}^{\prime}, f_{z^{\prime}}^{\prime}\right)$, where subscripts with commas denote differentiation with respect to the relevant variables. Assuming that the flaps perform small-amplitude oscillations, the set of boundary conditions (b.c.'s) associated to the Laplace equation (1) can be linearised as shown in [19] thus yielding

$$
\Phi_{, t^{\prime} t^{\prime}}^{\prime}+g \Phi_{, z^{\prime}}^{\prime}=0, \quad z^{\prime}=0
$$

for the kinematic-dynamic b.c. on the linearised free-surface,

$$
\Phi_{, z^{\prime}}^{\prime}=0, \quad z=-h^{\prime}
$$

for the no-flux condition at the bottom and finally

$$
\Phi_{, \hat{n}}^{\prime}=V_{m \hat{n}}^{\prime}, \quad \text { on } \partial \mathcal{L}_{m}^{\prime}, m=1,2, \ldots, M
$$

for the kinematic b.c. on the flaps. In the latter expression, $V_{m \hat{n}}^{\prime}$ is the component of the $m$ th-flap velocity along the normal $\hat{n}=\left(n_{x^{\prime}}, n_{y^{\prime}}, n_{z^{\prime}}\right)$ directed out of the fluid on the boundary $\partial \mathcal{L}_{m}^{\prime}[12]$. Hence for pitching motion about the $y^{\prime}$ axis, expression (4) yields

$$
\Phi_{, x^{\prime}}^{\prime}=-\theta_{m, t^{\prime}}^{\prime}\left(t^{\prime}\right)\left(z^{\prime}+h^{\prime}-c_{m}^{\prime}\right) H\left(z^{\prime}+h^{\prime}-c_{m}^{\prime}\right), \quad m=1,2, \ldots M,
$$

along the flap sides normal to the $x^{\prime}$ axis, where the Heaviside step function $H$ assures absence of flux through the bottom foundation, and

$$
\Phi_{, y^{\prime}}^{\prime}=0, \quad m=1,2, \ldots M
$$

along the flap sides normal to the $y^{\prime}$ axis.

\subsection{Body motion and wave-power absorption}

Once the velocity potential $\Phi^{\prime}$ is known, the flap dynamics can be fully characterised by solving the following system of Newton's laws of motion (see also [22]):

$$
\begin{array}{r}
I_{m}^{\prime} \theta_{m, t^{\prime} t^{\prime}}^{\prime}\left(t^{\prime}\right)+C_{m}^{\prime} \theta_{m}^{\prime}\left(t^{\prime}\right)+\eta_{m}^{\prime} \theta_{m, t^{\prime}}^{\prime}\left(t^{\prime}\right)= \\
-\rho \int_{\partial \mathcal{L}_{m}^{\prime}} \Phi_{, t^{\prime}}^{\prime}\left(x^{\prime}, y^{\prime}, z^{\prime}, t^{\prime}\right)\left[n_{x^{\prime}}\left(z^{\prime}+h^{\prime}-c_{m}^{\prime}\right)+n_{z^{\prime}} x^{\prime}\right] d S^{\prime}, m=1, \ldots, M .
\end{array}
$$

The latter impose the equilibrium of the inertial, linear and damping actions (left-hand side) with the hydrodynamic torque (right-hand side), for each 
flap. In (7), $I_{m}^{\prime}, C_{m}^{\prime}$ and $\eta_{m}^{\prime}$ are, respectively, the moment of inertia, the buoyancy torque and the rate of power take-off (PTO) of the $m$ th flap, given by the system manufacturer, $\rho$ is water density. Once the system of ordinary differential equations (7) is solved in terms of the pitching angles $\theta_{m}^{\prime}\left(t^{\prime}\right)$, $m=1,2, \ldots, M$, the performance of the array can be analysed by calculating the amplitude factor of each flap and the total power output. The $m$ th amplitude factor is defined as the ratio between the maximum horizontal displacement of the $m$ th flap at the free-surface in a period and the amplitude of the incident wave, that is

$$
A_{m}^{F}=\frac{\tan \left[\max _{T^{\prime}} \theta_{m}^{\prime}\right]\left(h^{\prime}-c_{m}^{\prime}\right)}{A_{I}^{\prime}} .
$$

According to (8), the larger the amplitude factor, the larger the amount of incoming wave energy which is converted into pitching motion by each flap, for given non-zero PTO damping. Finally, the average power absorbed by the system in a period is calculated by summing the contribution of each single flap (see [22]):

$$
P^{\prime}=\frac{1}{T^{\prime}} \sum_{m=1}^{M} \int_{0}^{T^{\prime}}\left(\eta_{m}^{\prime} \theta_{m, t^{\prime}}^{\prime}\right) \theta_{m, t^{\prime}}^{\prime} d t^{\prime} .
$$

In the following, the system of equations (1)-(6) will be solved with either a semi-analytical model or a fully numerical model. Then the dynamics of the flaps and the performance of the system will be analysed according to equations (7)-(9). The semi-analytical model is based on the simplifying assumption that all flaps are aligned on the $y^{\prime}$ axis. Such assumption restricts the applicability of the model to idealised cases, but allows to gain a fundamental insight into the dynamics of the system which could be overlooked by relying only on numerical calculations. On the other hand, the numerical model is not restricted by such constraints and can be used to further investigate practical scenarios where the semi-analytical model cannot be applied. In this sense, the two models complement well and together provide an essential tool for an in-depth analysis of the system in the linear regime.

\subsection{Semi-analytical solution}

Consider an in-line system of flaps aligned along the $y^{\prime}$ axis. In this case, the analytical approach of Adamo \& Mei [29] for an array of neighbouring 


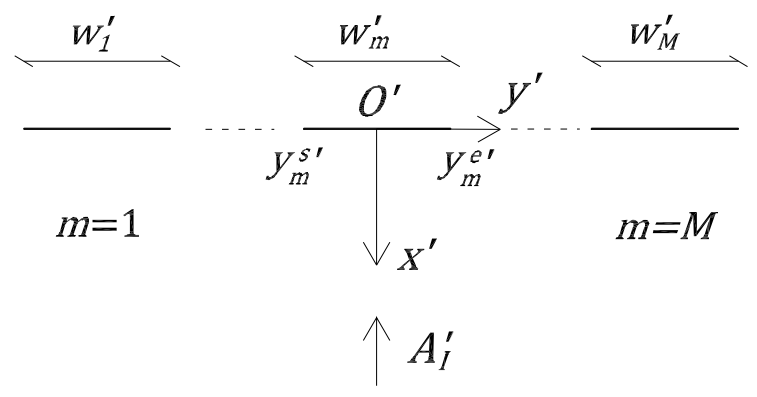

Figure 2: Geometry of an array made by $M$ inline OWSCs. The thin-plate approximation $[19,20,22]$ is applied to calculate the velocity potential in normally incident waves.

flood gates can be extended and combined with the integral formulation of Renzi \& Dias [22] for a single OWSC in the open ocean. Such procedure allows to obtain a new semi-analytical solution of the problem. Assuming that the characteristic length scale of the system $b^{\prime}$ (e.g. the width of the largest flap) is much larger than the thickness of each device, i.e. $b^{\prime} \gg a_{m}^{\prime}$, the latter can be considered immaterial for calculating the potential $\Phi^{\prime}$ (thinplate approximation, see also $[19,22,29])$. As a consequence, each flap is represented in the $\left(x^{\prime}, y^{\prime}\right)$ plane as a line lying on the $y^{\prime}$ axis, as shown in figure 2. Now let $y_{m}^{s \prime}$ and $y_{m}^{e \prime}$ denote, respectively, the coordinates of the start and end points of the $m$ th flap on the $y^{\prime}$ axis, with $y_{m}^{s}{ }^{\prime}<y_{m}^{e}{ }^{\prime}<y_{(m+1)}^{s}{ }^{\prime}<$ $\cdots<y_{M}^{e}{ }^{\prime}$. Hence the $m$ th-flap width is $w_{m}^{\prime}=y_{m}^{e \prime}-y_{m}^{s \prime}>0$ and the relevant border reduces to

$\partial \mathcal{L}_{m}^{\prime}=\left\{\left(x^{\prime}, y^{\prime}, z^{\prime}\right): x^{\prime}= \pm 0, y^{\prime} \in\left(y_{m}^{s \prime}, y_{m}^{e \prime}\right), z^{\prime} \in\left(-h^{\prime}, 0\right)\right\}, m=1,2, \ldots, M$

Then introduce the following nondimensional variables

$$
(x, y, z)=\left(x^{\prime}, y^{\prime}, z^{\prime}\right) / b^{\prime}, t=\sqrt{g / b^{\prime}} t^{\prime}, \Phi=\Phi^{\prime}\left(\sqrt{g b^{\prime}} A^{\prime}\right)^{-1}, \theta_{m}=\left(b^{\prime} / A^{\prime}\right) \theta_{m}^{\prime}
$$

and constants

$$
\left(c_{m}, y_{m}^{s}, y_{m}^{e}, w_{m}, h\right)=\left(c_{m}^{\prime}, y_{m}^{s}{ }^{\prime}, y_{m}^{e \prime}, w_{m}^{\prime}, h^{\prime}\right) / b^{\prime}, A_{I}=A_{I}^{\prime} / A^{\prime} .
$$

In (11) and (12) $A^{\prime}$ is the amplitude scale of the incident wave. This must be much smaller than the length scale of the system to assure that the converters perform small-amplitude oscillations, i.e. $A^{\prime} \ll b^{\prime}$ (see also [19, 22]). Let us 
also consider time-harmonic oscillations of radian frequency $\omega=\omega^{\prime} \sqrt{b / g}=$ $2 \pi / T$ and factor time out of the time-dependent variables, such as

$$
\Phi(x, y, z, t)=\Re\left\{\phi(x, y, z) e^{-i \omega t}\right\}, \theta_{m}(t)=\Re\left\{\Theta_{m} e^{-i \omega t}\right\}, m=1,2, \ldots, M,
$$

where $\Re$ denotes the real part, $\phi$ is the complex spatial potential and $\Theta_{m}$ the complex amplitude of rotation of the $m$ th flap. Given the linearity of the system, position (13) assumes that all flaps oscillate with the same frequency $\omega$ (this excludes second-order resonant effects, see [25, 31, 32]), but with different phases $\epsilon_{m}=\arg \left(\Theta_{m}\right)$. Substituting the nondimensional variables (11) together with the factorisation (13) into the governing equations (1)-(6) yields, respectively,

$$
\nabla^{2} \phi=0
$$

in the fluid domain for the Laplace equation (1),

$$
\phi_{, z}-\omega^{2} \phi=0, \quad z=0
$$

for the b.c. on the free surface (2),

$$
\phi_{, z}=0, \quad z=-h
$$

for the no-flux condition at the bottom (3) and finally

$$
\phi_{, x}=\Omega_{m}\left(z+h-c_{m}\right) H\left(z+h-c_{m}\right), \quad \text { on } \partial \mathcal{L}_{m}, m=1,2, \ldots, M
$$

for the kinematic condition on the flaps (5). In expression (17), $\Omega_{m}=i \omega \Theta_{m}$ for the sake of brevity. Now assume for $\phi$ the same decomposition argument as that of Adamo \& Mei [29] (see eq. 2.4), for which

$$
\phi=\phi^{S}+\sum_{\alpha=1}^{M} \Omega_{\alpha} \phi^{(\alpha)} .
$$

In the latter, $\phi^{S}=\phi^{I}+\phi^{D}$ is the scattering potential, solution of the problem in which the flaps are all held fixed in incoming waves. In turn $\phi^{S}$ is the sum of the incident wave potential

$$
\phi^{I}=-i \frac{A_{I}}{\omega} \frac{\cosh k(z+h)}{\cosh k h} e^{-i k x}
$$

176 and the unknown diffraction potential $\phi^{D}$. In (19) $k$ is the wavenumber 177 given by the well-known dispersion relation $\omega^{2}=k \tanh k h$. Finally, $\phi^{(\alpha)}$ is 


$$
\left\{\begin{array}{c}
\phi_{, x}^{D} \\
\phi_{, x}^{(\alpha)}
\end{array}\right\}=\left\{\begin{array}{c}
-\phi_{, x}^{I} \\
\left(z+h-c_{\alpha}\right) H\left(z+h-c_{\alpha}\right) \delta_{\alpha m}
\end{array}\right\} \text {, on } \partial \mathcal{L}_{m}, m=1, \ldots, M .
$$

the solution of the unit radiation potential, where the $\alpha$ th flap moves with $\Omega_{\alpha}=1$ while all the other flaps are at rest. Note that $\phi^{I}$ (19) models a plane wave field normally incident on the system. Oblique incidence will not be investigated here because of two main reasons: (a) the system is to be deployed in the nearshore, where wave fronts are almost parallel to the shoreline due to refraction and (b) in case of oblique incidence at an angle $\beta \neq 0$ with the $x$ axis, the power captured by the OWSC would decrease almost as $\cos ^{2} \beta$, as shown by Whittaker et al. [33]. Hence oblique incidence is undesirable and is usually avoided by orienting the flaps along the direction of predominant incidence. This allows to minimise the reduction in power capture due to obliquely incident waves (e.g. about $3 \%$ in random seas for the Oyster device, see [33]) which can be neglected without consequences. In order to solve the system (14)-(17), the linearised potentials introduced in the decomposition (18) must satisfy the governing equation

$$
\nabla^{2}\left\{\begin{array}{c}
\phi^{D} \\
\phi^{(\alpha)}
\end{array}\right\}=0
$$

in the fluid domain, together with the boundary conditions

$$
\begin{aligned}
\left\{\begin{array}{c}
\phi_{, z}^{D} \\
\phi_{, z}^{(\alpha)}
\end{array}\right\}-\omega^{2}\left\{\begin{array}{c}
\phi^{D} \\
\phi^{(\alpha)}
\end{array}\right\}=0, & z=0, \\
\left\{\begin{array}{c}
\phi_{, z}^{D} \\
\phi_{, z}^{(\alpha)}
\end{array}\right\}=0, & z=-h,
\end{aligned}
$$

In the latter, $\delta_{\alpha m}$ is the Kronecker delta, $m$ indicates any flap and $\alpha$ the moving flap. The first scalar equation of (23) requires the scattering potential $\phi^{S}=\phi^{I}+\phi^{D}$ to satisfy a no-flux condition on all the flaps. Meanwhile, the second scalar equation of (23) requires the radiation potential $\phi^{(\alpha)}$ to satisfy a no-flux condition on all the non-moving flaps, for which $m \neq \alpha$, and a kinematic b.c. on the moving flap $m=\alpha$, for all $\alpha=1, \ldots, M$ in succession. Now separate the vertical dependence of the spatial potentials by using the decomposition

$$
\left\{\begin{array}{c}
\phi^{D} \\
\phi^{(\alpha)}
\end{array}\right\}=\sum_{n=0}^{\infty}\left\{\begin{array}{c}
\varphi_{n}^{D} \\
\varphi_{n}^{\alpha}
\end{array}\right\} Z_{n}(z)
$$


where the $\varphi_{n}^{D}(x, y)$ and $\varphi_{n}^{\alpha}(x, y)$ are, respectively, two-dimensional (2D) diffraction and radiation potentials in the $(x, y)$ plane. In $(24)$ the $Z_{n}$ are the well-known vertical eigenmodes

$$
Z_{n}(z)=\frac{\sqrt{2} \cosh \kappa_{n}(z+h)}{\left(h+\omega^{-2} \sinh ^{2} \kappa_{n} h\right)^{1 / 2}},
$$

where $\kappa_{0}=k, \kappa_{n}=i k_{n}$ and the $k_{n}$ are the real solutions of the dispersion relation

$$
\omega^{2}=-k_{n} \tan k_{n} h, \quad n=1,2, \ldots
$$

Substitution of the series expansion (24) into (20)-(23) and usage of the orthogonality property

$$
\int_{-h}^{0} Z_{n}(z) Z_{m}(z) d z=\delta_{n m}
$$

for the vertical eigenfunctions (25) yields the following system of equations for the diffraction and radiation potentials:

$$
\left(\nabla^{2}+\kappa_{n}^{2}\right)\left\{\begin{array}{c}
\varphi_{n}^{D} \\
\varphi_{n}^{\alpha}
\end{array}\right\}=0
$$

1 in the 2D fluid domain and

$$
\left\{\begin{array}{c}
\varphi_{n, x}^{D} \\
\varphi_{n, x}^{\alpha}
\end{array}\right\}=\left\{\begin{array}{c}
A_{I} d_{n} \\
f_{n \alpha} \delta_{\alpha m}
\end{array}\right\}, \quad x= \pm 0, y \in\left(y_{m}^{s}, y_{m}^{e}\right)
$$

on each flap $m=1,2, \ldots, M$. In expression $(29)$

$$
\begin{aligned}
d_{n} & =\frac{k\left(h+\omega^{-2} \sinh ^{2} k h\right)^{1 / 2}}{\sqrt{2} \omega \cosh k h} \delta_{n 0}, \\
f_{n \alpha} & =\sqrt{2} \frac{\kappa_{n}\left(h-c_{\alpha}\right) \sinh \kappa_{n} h+\cosh \kappa_{n} c_{\alpha}-\cosh \kappa_{n} h}{\kappa_{n}^{2}\left(h+\omega^{-2} \sinh ^{2} \kappa_{n} h\right)^{1 / 2}}
\end{aligned}
$$

are real values depending on the system parameters. Finally, it is required that both $\varphi_{n}^{D}$ and $\varphi_{n}^{\alpha}$ be outgoing disturbances as $x, y \rightarrow \infty$. The system of equations (28)-(31) can be solved by extending to a finite array of flaps the method developed by Renzi \& Dias [22] for a single flap in the open ocean. The procedure is detailed in Appendix A and briefly summarised 
here. Application of Green's integral theorem to a large circle enclosing all the flaps and usage of the b.c.'s (29) yields a hypersingular integral equation for each of the $2 \mathrm{D}$ potentials. The system is hence carefully de-singularised by recurring to a series expansion in terms of the Chebyshev polynomials of the second kind (see Appendix A for details). As a result, the spatial diffraction potential can be written as

$$
\begin{aligned}
\phi^{D}(x, y, z) & =\frac{-i A_{I}}{8} k x Z_{0}(z) \sum_{\beta=1}^{M} w_{\beta} \sum_{p=0}^{P} a_{0 \beta p} \int_{-1}^{1}\left(1-u^{2}\right)^{1 / 2} \\
\times & U_{p}(u) \frac{H_{1}^{(1)}\left[k \sqrt{x^{2}+\left(y-1 / 2\left(u_{\beta}+w_{\beta} u\right)\right)^{2}}\right]}{\sqrt{x^{2}+\left(y-1 / 2\left(u_{\beta}+w_{\beta} u\right)\right)^{2}}} d u
\end{aligned}
$$

where $u_{\beta}=y_{\beta}^{e}+y_{\beta}^{s}, w_{\beta}=y_{\beta}^{e}-y_{\beta}^{s}, H_{1}^{(1)}$ is the outgoing Hankel function of the first kind and first order, $U_{p}$ is the Chebyshev polynomial of the second kind and order $p \in \mathbb{N}$ and finally the $a_{0 \beta p}$ are complex coefficients which assure that the diffraction potential satisfies the b.c. on the flaps (29). Such coefficients are determined by solving a system of linear equations with a numerical collocation scheme (see again Appendix A). Therefore the solution (32) is semi-analytical. Note that the diffraction potential $\phi^{D}(32)$ depends only on the 0th-order vertical mode. This is due to a solvability condition on the coupled radiation-diffraction problem which excludes higher-order vertical eigenmodes in $\phi^{D}$ (for details see Appendix A and [19]). The unit radiation potential for the $\alpha$ th plate is given by

$$
\begin{aligned}
\phi^{(\alpha)}(x, y, z) & =\frac{-i}{8} \sum_{n=0}^{\infty} \kappa_{n} x Z_{n}(z) \sum_{\beta=1}^{M} w_{\beta} \sum_{p=0}^{P} b_{n \alpha \beta p} \int_{-1}^{1}\left(1-u^{2}\right)^{1 / 2} \\
& \times U_{p}(u) \frac{H_{1}^{(1)}\left[\kappa_{n} \sqrt{x^{2}+\left(y-1 / 2\left(u_{\beta}+w_{\beta} u\right)\right)^{2}}\right]}{\sqrt{x^{2}+\left(y-1 / 2\left(u_{\beta}+w_{\beta} u\right)\right)^{2}}} d u .
\end{aligned}
$$

In the latter, the $b_{n \alpha \beta p}$ are complex coefficients which assure that the radiation potential satisfies the boundary condition on the flaps (29). Such coefficients are again determined by solving a system of linear equations with a numerical collocation scheme, so that (33) is semi-analytical. 
In order to solve the problem fully, the pitching angles $\Theta_{m}$ in the decomposition (13) must now be determined. This can be done by considering the equations of motion of the system (7), on one flap - say the $\beta$ th flap - at a time. Substituting the factorisation (13) for the total potential and the pitching angles into (7), together with (18), (19), (24), (A.3), (A.5) and (A.9), yields after some lengthy algebra

$$
\left[-\omega^{2}\left(I_{\beta}+\mu_{\beta \beta}\right)+C_{\beta}-i \omega\left(\nu_{\beta \beta}+\eta_{\beta}\right)\right] \Theta_{\beta}-\sum_{\alpha=1}^{M},\left(\omega^{2} \mu_{\alpha \beta}+i \omega \nu_{\alpha \beta}\right) \Theta_{\alpha}=F_{\beta},
$$

for $\beta=1, \ldots, M$, where the potential inside the surface integral in (7) has been evaluated by using the thin-plate approximation (10). In (34), $I_{\beta}=$ $I_{\beta}^{\prime} /\left(\rho b^{\prime 5}\right), C_{\beta}=C_{\beta}^{\prime} /\left(\rho g b^{\prime 4}\right)$ and $\eta_{\beta}=\eta_{\beta}^{\prime} /\left(\rho b^{\prime 4} \sqrt{g b^{\prime}}\right)$ are, respectively, the nondimensional moment of inertia, buoyancy torque and PTO damping of the $\beta$ th flap and $\sum^{\prime}$ indicates that the summation must exclude the term $\alpha=\beta$. Furthermore, in (34)

$$
F_{\beta}=-\frac{i \pi}{4} A_{I} w_{\beta} \omega f_{0 \beta} a_{0 \beta 0}
$$

is the nondimensional exciting torque acting on the $\beta$ th flap when all the flaps are fixed in incident waves, while

$$
\mu_{\alpha \beta}=\frac{\pi w_{\beta}}{4} \sum_{n=0}^{\infty} f_{n \beta} \Re\left\{b_{n \alpha \beta 0}\right\}
$$

and

$$
\nu_{\alpha \beta}=\frac{\pi w_{\beta}}{4} \omega \sum_{n=0}^{\infty} f_{n \beta} \Im\left\{b_{n \alpha \beta 0}\right\}
$$

are, respectively, the nondimensional added mass and radiation damping due to pressure distribution on flap $\beta$ when flap $\alpha$ is moving (see for example $[8,29]$ ). Due to the reciprocity relations of Appendix B it is straightforward to obtain the well-known results $[8,12,29,34]$

$$
\mu_{\alpha \beta}=\mu_{\beta \alpha}, \quad \nu_{\alpha \beta}=\nu_{\beta \alpha} .
$$

The latter relations are used in this paper to test the accuracy of the numerical computations in the semi-analytical model. The system of equations 
(34) allows to obtain the sought complex amplitude of rotation $\Theta_{\beta}$ for each flap $\beta=1, \ldots, M$. Physically, the first term on the left-hand side of (34) represents the direct dynamic influence of the $\beta$ th flap on itself, while the other terms represent the influence of all the other flaps on flap $\beta$. Finally, note that in (34) the buoyancy restoring torque acting on the $\beta$ th flap is fully accounted for by the term $C_{\beta}$ [19]. In other words, the thin-plate approximation is applied only to the velocity potential and does not affect the buoyancy property of the flaps [29]. By introducing the matrix components

$$
\begin{gathered}
{[\boldsymbol{\Theta}]_{\beta}=\Theta_{\beta},[\mathbf{I}]_{\alpha \beta}=I_{\alpha} \delta_{\alpha \beta},[\mathbf{M}]_{\alpha \beta}=\mu_{\alpha \beta},[\mathbf{C}]_{\alpha \beta}=C_{\alpha} \delta_{\alpha \beta}} \\
{[\mathbf{N}]_{\alpha \beta}=\nu_{\alpha \beta},[\mathbf{H}]_{\alpha \beta}=\eta_{\alpha} \delta_{\alpha \beta},[\mathbf{F}]_{\beta}=F_{\beta},}
\end{gathered}
$$

the system (34) can also be rewritten in the matrix form

$$
\left[-\omega^{2}(\mathbf{I}+\mathbf{M})+\mathbf{C}-i \omega(\mathbf{N}+\mathbf{H})\right]^{\mathrm{T}} \boldsymbol{\Theta}=\mathbf{F},
$$

which is more convenient for implementation in numerical routines (see also [8]). Finally, once the $\Theta_{\beta}$ are known from (34) or (40), the average absorbed power over a cycle is determined by substituting the second of (13) together with the physical scales of (11) into (9), which then yields

$$
P^{\prime}=\frac{\omega^{\prime 2}}{2} \sum_{\beta=1}^{M} \eta_{\beta}^{\prime}\left|\Theta_{\beta}^{\prime}\right|^{2} .
$$

\subsection{Fully numerical solution}

A finite-element model (FEM) based on the software COMSOL Multiphysics is applied to solve the wave-induced flow, coupled together with a numerical solver of the global dynamic equations of motion (7). The latter allows to calculate the movement of the flaps. For problems in fluid-structure interaction, usage of the boundary-element method (BEM) is also a viable option [see for example 26]. However, often BEM codes are significantly less efficient than volume-discretisation methods. This happens since BEM codes usually employ fully populated matrices. As a consequence, the storage requirements and computational time for a BEM code grows according to the square of the problem size. On the other hand, FEM codes employ banded matrices, whose storage requirements usually grow linearly with the problem size.

Unlike the semi-analytical model of $\S 2.3$, the numerical model is able to reproduce virtually any array layout, for arbitrary angle of incidence of the 

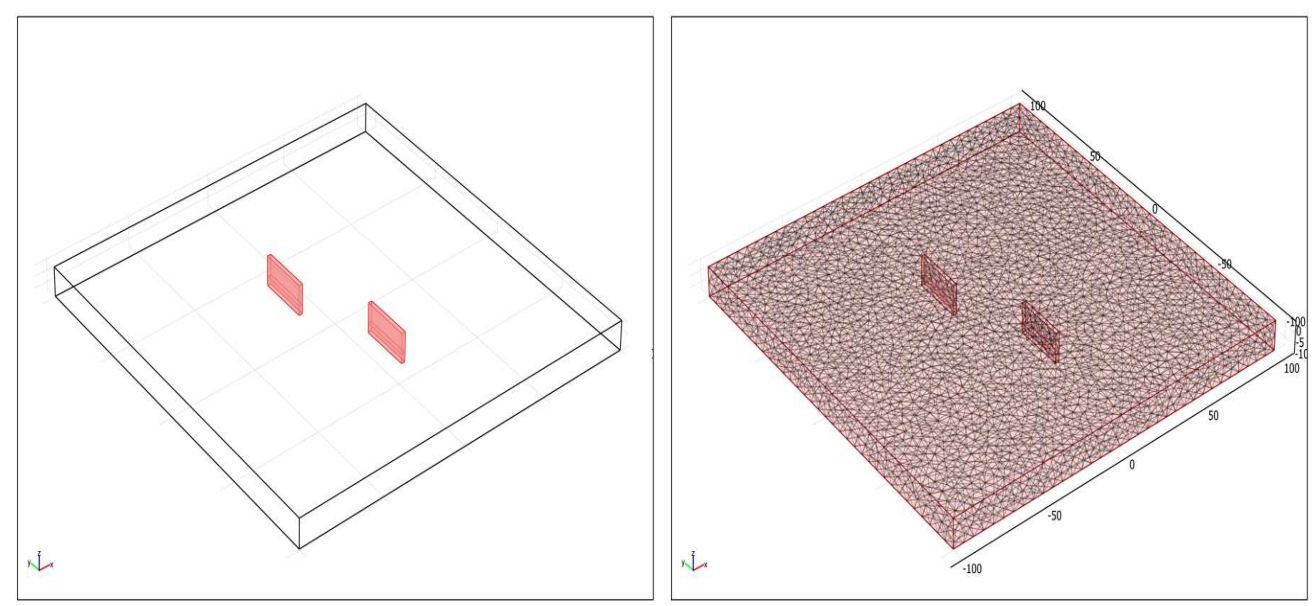

Figure 3: Left panel: sketch of the computational domain of the numerical model for an array of two staggered OWSCs (see $\S 4$ for parameters). Right panel: same model with the tetrahedral mesh elements represented.

incoming waves, indeed at larger computational cost (see §2.5). The finiteelement method is used which ensures an excellent reproduction of domains with complex geometries. Tetrahedral elements are used to model threedimensional bodies, as shown in figure 3. Let us define a reference frame by considering $x^{\prime}$ the longitudinal horizontal direction, $z^{\prime}$ the vertical direction, pointing upwards, and $y^{\prime}$ the remaining horizontal direction originating from the center of the numerical domain. Similar to the semi-analytical model, the numerical model is based on the assumptions of irrotational flow, inviscid fluid, small-amplitude waves and small movement of the flaps with respect to the wavelength and to the water depth. As a consequence, the flow field is governed again by the Laplace equation (1), the kinematic-dynamic boundary condition on the free surface (2), the no-flux condition at the bottom (3) and the kinematic condition on the wet boundary of the flaps (4), conveniently re-written in the form (see [35])

$$
\hat{n} \cdot \nabla \Phi^{\prime}=n_{x^{\prime}} r^{\prime} \theta_{, t^{\prime}}^{\prime}
$$

In the latter, $n_{x^{\prime}}$ represents the $x^{\prime}$ component of the normal to the flap surfaces at rest and $r^{\prime}$ is the distance between any point on the flap sides and the hinge axis, for each flap. The flaps are initially considered at rest position: $\theta_{m}^{\prime}(0)=0, m=1, \ldots, M$. An approximate radiation condition including a source term for generating the desired incoming waves is imposed at the open 
generation boundary (left of the domain in figure 3), which also allows the waves reflected back by the device to freely leave the computational domain:

$$
\hat{n} \cdot \nabla \Phi^{\prime}=-\frac{\cos \alpha_{n}}{c^{\prime}} \Phi_{t^{\prime}}^{\prime}+\frac{2 A_{I}^{\prime} g}{\omega^{\prime}} k^{\prime} \frac{\cosh k^{\prime}\left(z^{\prime}+h^{\prime}\right)}{\cosh k^{\prime} z^{\prime}} \cos \omega^{\prime} t^{\prime} .
$$

In the latter, $c^{\prime}=\omega^{\prime} / k^{\prime}$ is the linear phase speed, $k^{\prime}$ is the wavenumber solution of $\omega^{\prime 2}=g k^{\prime} \tanh k^{\prime} h^{\prime}$ and $\alpha_{n}$ is the angle formed by the incoming wave direction with the outgoing normal to the considered boundary. At the remaining lateral boundaries, a sink term is imposed, obtained by using a mathematical formulation $[36,37]$ which allows the waves propagating outwards to freely exit the computational domain:

$$
\hat{n} \cdot \nabla \Phi^{\prime}=-\frac{\cos \alpha_{n}}{c^{\prime}} \Phi_{, t^{\prime}}^{\prime}
$$

The solution of the flow field is then used to fully determine the flap kinematics by solving the system of equations of motion (7). Once the pitching angle $\theta_{m}^{\prime}$ is determined for each flap $m=1, \ldots, M$ at each time step, the performance of each OWSC is evaluated by calculating the amplitude factor (8) and the absorbed power (9). A description of the numerical procedure adopted in this paper is offered in Appendix C.

\subsection{Computational aspects}

The semi-analytical model of $\S 2.3$ has been implemented on a parallelised code on Mathematica ${ }^{\circledR} 8$ (see for example [38]), able to evaluate the exciting torque (35), the added inertia torque (36), the radiation damping (37), the amplitude factor (8) and the total extracted power (41) by each flap in the array at given periods of the incident wave. For the 3-flap array examined in $\S 3$, the first three vertical eigenmodes and six Chebyshev polynomials i.e. a $(3,6)$ configuration - were sufficient to achieve a relative error $O\left(10^{-3}\right)$ with respect to a $(4,8)$ configuration whose computational time was almost two and a half times larger. For a typical array of three flaps, an average computational time of $28 \mathrm{~s}$ was sufficient to determine the abovementioned parameters for given period of the incident wave on a high-speed computer equipped with an i7 $3.40 \mathrm{GHz}$ CPU and 16 GB RAM.

For the numerical model of $\S 2.4$, possible symmetries occurring in the array configuration should always be looked for and exploited in order to reduce the computational time. As an example, for in-line arrays like the ones discussed in $\S 3$, symmetry of the problem about $y^{\prime}=0$ allows computations 
to be conveniently undertaken for half the physical domain only. Such a procedure is indeed not applicable to more complicated layouts, like the staggered array modelled in $\S 4$ (see also figure 3 ), where the model must be solved for the whole domain. For the most demanding simulation undertaken in this paper, the number of elements and degrees of freedom used were about 550,000 and 120,000 respectively. Typically, long wave lengths require a larger computational time but a coarse mesh, while short waves require a smaller domain but a fine mesh. In all cases, the flume length should be at least 3 times the wave length to assure that there is enough space for waves to develop and then leave the domain. On average, the computational time for $500 \mathrm{~s}$ of real-time simulation was about 1 hour on a high-speed computer equipped with an i7 2.67 GHz CPU and 12GB RAM.

\section{Discussion}

In this section, the semi-analytical model of $\S 2.3$ and the numerical model of $\S 2.4$ will be used to investigate the dynamics of a finite array of OWSCs. Results will be validated showing good agreement between the two different approaches in describing the hydrodynamic behaviour of the system. Furthermore, the semi-analytical model will be used to investigate the dependence of the system on its main parameters, i.e. the number of flaps and the flap spacing. For the first time, a near-resonant phenomenon is shown to occur for an in-line array of OWSCs.

\subsection{Validation}

The general analytical and numerical approaches presented in this paper have been already validated with experimental data for a single flap in a channel [19] and with other numerical results for a single flap in the open ocean $[22,23]$. In this section, results of the semi-analytical and numerical models for a finite array of OWSCs developed, respectively, in $\S 2.3$ and $\S 2.4$ are compared and validated for two different systems made by two and three in-line flaps.

\subsubsection{2-flap system}

First, consider a system of two identical in-line flaps $(1-2)$, each of width $w^{\prime}=26 \mathrm{~m}$ hinged at $c^{\prime}=4 \mathrm{~m}$ on an ocean of depth $h^{\prime}=13 \mathrm{~m}$. The thickness of the flaps is immaterial for calculating the velocity potential (but not the buoyancy torque, which is fully accounted for) in the semi-analytical model 

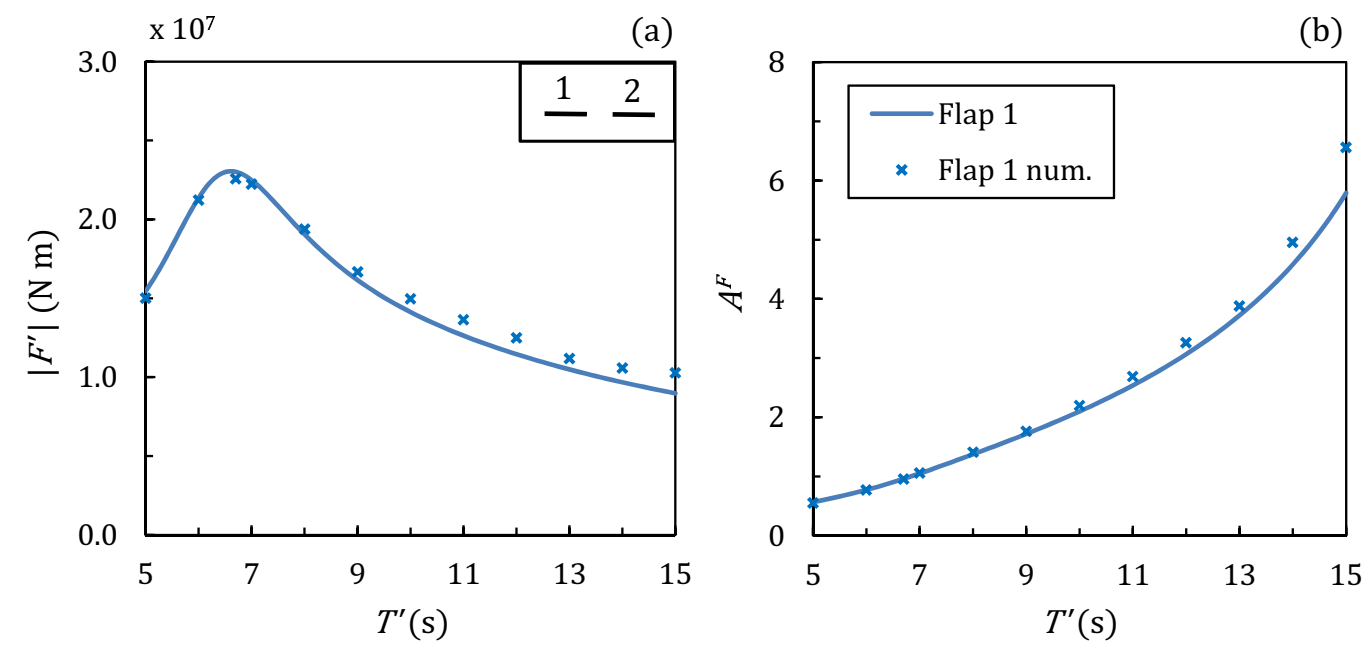

Figure 4: (a) Exciting torque magnitude in physical variables $\left|F^{\prime}\right|=\rho g A^{\prime} b^{\prime 3}|F|$ and (b) amplitude factor versus period of the incident wave calculated with the semi-analytical model (line) and the numerical model (ticks) for a two-flap system. In the semi-analytical model, $|F|$ is given by (35). In the numerical model, $\left|F^{\prime}\right|$ has been calculated directly by integrating the pressure field acting on the flaps held fixed in incident waves. The legend at the top right corner of (a) shows a sketch of the configuration (not in scale). Parameters are $w_{1}^{\prime}=w_{2}^{\prime}=26 \mathrm{~m}, c_{1}^{\prime}=c_{2}^{\prime}=4 \mathrm{~m}, s^{\prime}=30 \mathrm{~m}$ and $h^{\prime}=13 \mathrm{~m}$. Waves of amplitude $A_{I}^{\prime}=1 \mathrm{~m}$ are normally incident on the system. Due to linearity and symmetry of the system, $F_{1}^{\prime}=F_{2}^{\prime}$ and $A_{1}^{F}=A_{2}^{F}$.

and is $a^{\prime}=2 \mathrm{~m}$ in the numerical model. Such dimensions resemble those of the Oyster $800^{\mathrm{TM}}$ OWSC manufactured by APL [11]. The gap between the flaps is $s^{\prime}=30 \mathrm{~m}$, which gives $s=s^{\prime} / w^{\prime}=O(1)$. The PTO coefficient is the same for both flaps, i.e. $\eta_{1}^{\prime}=\eta_{2}^{\prime}$, and is chosen in order to maximise the total power output (41) in the semi-analytical model, for each given period of the incident wave. The inertia and buoyancy torque coefficients, respectively $I$ and $C$, are equal for all flaps and have been obtained by private communication with APL. Consider a monochromatic wave of amplitude $A_{I}^{\prime}=1 \mathrm{~m}$ normally incident on the farm. Due to the linearity of the system and the symmetry of the layout, the flaps are expected to oscillate at unison. Hence knowing the behaviour of only one flap is sufficient to characterise the behaviour of the whole system. Figure 4 shows a sketch of the system layout together with the plots of the exciting torque magnitude $\left|F_{1}^{\prime}\right|=\left|F_{2}^{\prime}\right|$ in physical variables and the amplitude factor $A_{1}^{F}=A_{2}^{F}$ for both the semianalytical model of $\S 2.3$ and the numerical model of $\S 2.4$. The agreement between the data sets is good at short periods and satisfactory at larger 
periods, where the influence of the thickness determines the numerical values to be slightly larger than the semi-analytical ones. With longer waves, in fact, the jump in potential across a thick flap is larger than that across a thin flap, to which a long wave passes almost as a uniform swelling [19]. This determines the exciting torque to be larger for a thick flap and justifies the slight difference between the semi-analytical and numerical results in figure 4. Figure 4(a) shows that the system attains the maximum exciting torque at small periods of the incident wave. This is a peculiar characteristic of the OWSC which is already known to occur for a single device in the open ocean $[18,22]$ and for an infinite array of OWSCs [20]. Results of figure 4(a) reveal that the OWSC maintains this fundamental feature also when working in a finite array. Furthermore, figure 4(b) shows that the amplitude factor of either flap increases monotonically with the period of the incident waves. Already for $T^{\prime} \geq 7 \mathrm{~s}, A^{F}$ is larger than 1 , which reveals that the system effectively converts the incident wave motion into pitching motion.

\subsubsection{3-flap system}

A similar analysis is now made for a more complex system of three inline flaps $(1-2-3)$ with same dimensions and spacing as above. The PTO coefficient is the same for all the flaps, i.e. $\eta_{1}^{\prime}=\eta_{2}^{\prime}=\eta_{3}^{\prime}$, and again is chosen in order to maximise the total power output (41) for each given period of the incident wave. A monochromatic wave of amplitude $A_{I}^{\prime}=1 \mathrm{~m}$ normally incident on the system is considered. Due to symmetry, the system of equations of motion (7) needs to be solved for flaps 1 and 2 only, with flap 3 mirroring flap 1. Figure 5 shows a sketch of the system layout together with the plots of the exciting torque magnitudes $\left|F_{1}^{\prime}\right|=\left|F_{3}^{\prime}\right|,\left|F_{2}^{\prime}\right|$ and the amplitude factors $A_{1}^{F}=A_{3}^{F}, A_{2}^{F}$ versus the period of the incident wave for both the semi-analytical and the numerical models. Again, the agreement between the data sets is good at short periods and satisfactory at larger periods, due to the influence of the flap thickness, for which the numerical values are slightly larger than the semi-analytical ones. The same qualitative comments made above for the two-flap array can be repeated in full also for the three-flap array. Having validated the models, we shall employ the semianalytical model to perform the parametric analysis of the system. The latter will reveal the existence of a near-resonant phenomenon for in-line arrays of OWSCs. 

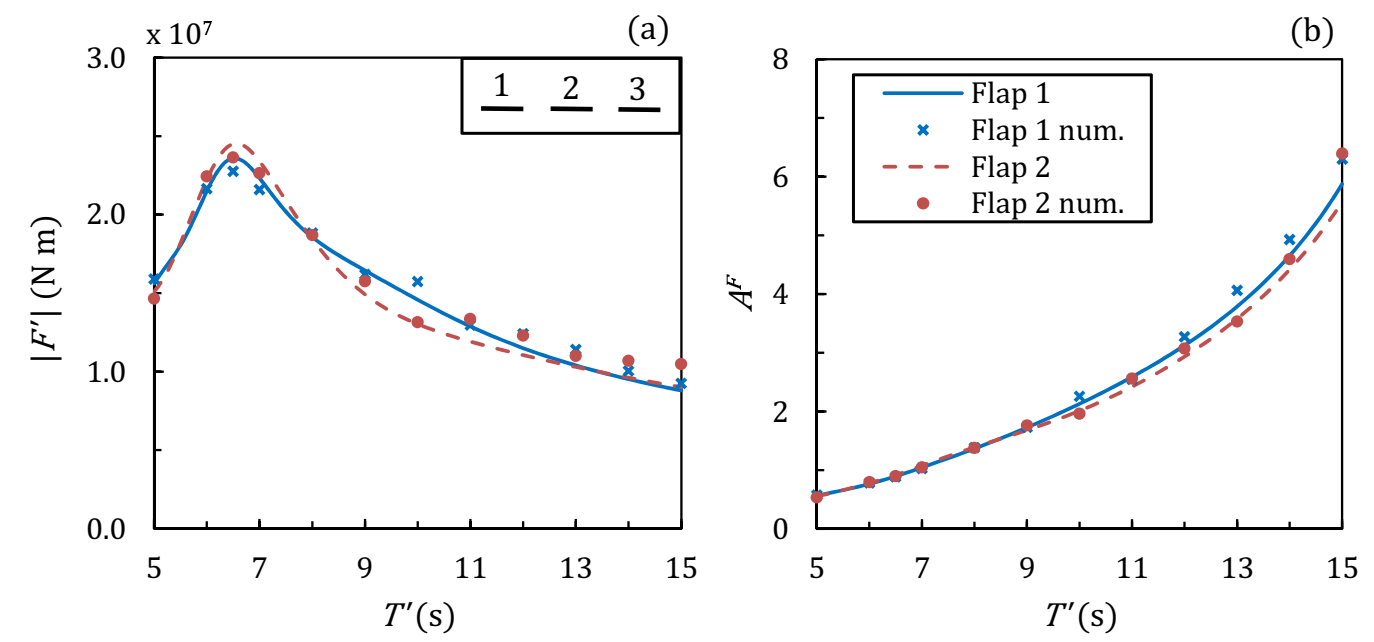

Figure 5: (a) Exciting torque magnitude in physical variables $\left|F^{\prime}\right|=\rho g A^{\prime} b^{\prime 3}|F|$ and (b) amplitude factor versus period of the incident wave calculated with the semi-analytical model and the numerical model for a three-flap system. The legend at the top right corner of (a) shows a sketch of the configuration (not in scale). Parameters are $w_{1}^{\prime}=w_{2}^{\prime}=w_{3}^{\prime}=$ $26 \mathrm{~m}, c_{1}^{\prime}=c_{2}^{\prime}=c_{3}^{\prime}=4 \mathrm{~m}$ and $h^{\prime}=13 \mathrm{~m}$. The gap is $s^{\prime}=30 \mathrm{~m}$ between all flaps. Waves of amplitude $A_{I}^{\prime}=1 \mathrm{~m}$ are normally incident on the farm. Due to linearity and symmetry of the system, $F_{1}^{\prime}=F_{3}^{\prime}$ and $A_{1}^{F}=A_{3}^{F}$.

\subsection{Parametric analysis}

A strong point of the analytical approach of $\S 2.3$ is that it easily allows to highlight the system dependence on its main parameters, i.e. the number of flaps and the flap spacing, at minimum computational cost.

\subsubsection{Influence of number of flaps and near resonance}

Figure 6 shows the behaviour of the exciting torque versus the period of the incident wave for several systems: a single flap in the open ocean [22], either flap in an array of two in-line devices, the central flap of an array of three in-line devices and any flap in an infinite in-line array [20]. In such layouts $w^{\prime}=26 \mathrm{~m}, s^{\prime}=30 \mathrm{~m}$ and $c^{\prime}=4 \mathrm{~m}$ for all the flaps, $h^{\prime}=13 \mathrm{~m}$ and waves of amplitude $A_{I}^{\prime}=1 \mathrm{~m}$ are normally incident. Note from figure 6 that the peak exciting torque for a finite array moves closer to that of an infinite array by increasing the number of elements. For an infinite array of OWSCs oscillating at unison, Renzi \& Dias [20] already showed that the maximum exciting torque occurs at one of the resonant periods of the system transverse modes. The latter correspond to the natural sloshing modes of a channel having the same width as the array spatial period [19]. In a finite array, on the 


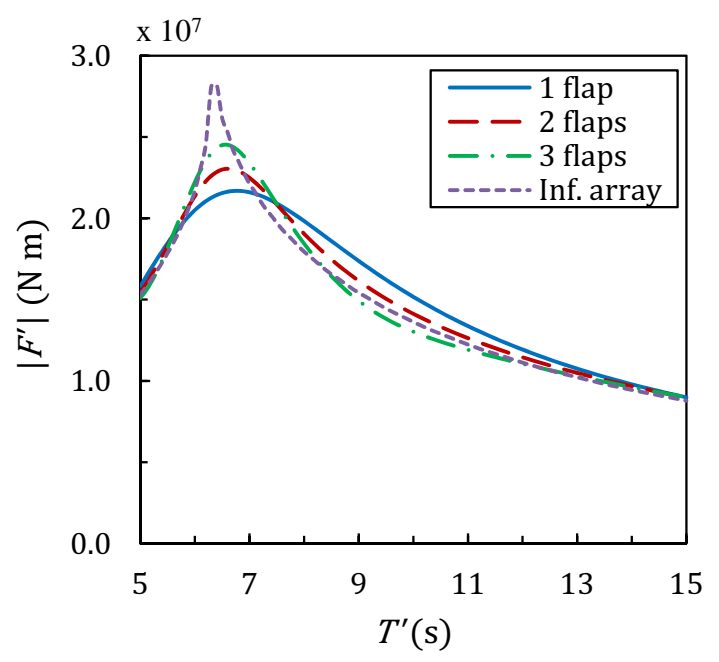

Figure 6: Exciting torque magnitude in physical variables versus period of the incident wave for a single flap (solid line), either flap of a two-flap system (dashed line), the central flap of a three-flap system (dash-dotted line) and any flap in an infinite array (dotted line). In all layouts $w^{\prime}=26 \mathrm{~m}, c^{\prime}=4 \mathrm{~m}, s^{\prime}=30 \mathrm{~m}$ for all flaps and $h^{\prime}=13 \mathrm{~m}$. Waves of amplitude $A_{I}^{\prime}=1 \mathrm{~m}$ are normally incident on the systems. Values are calculated with the semi-analytical model of $\S 2.3$ for the finite arrays and with the semi-analytical model of Renzi \& Dias [20] for the infinite array.

other hand, the increased energy leakage associated to the radial spreading of waves towards infinity attenuates such resonant behaviour (see also [39] for a similar mechanism in wave-structure interaction). As a result, the maximum torque for a finite array is lower than the corresponding infinite array value the attenuation being more significant for less populated arrays - and is also shifted towards longer waves (see again figure 6). The occurrence of peaks in the exciting torque acting on the elements of a finite array at periods close to the transverse-mode resonance of an infinite array is a near-resonant mechanism. This phenomenon has similar effects - yet different nature [27] - compared with the near-trapping phenomenon observed in previous work $[27,30,40]$, for which frequency dependent resonance yields peaks in the hydrodynamic actions on the elements of a stationary array of cylinders. Indeed, for a finite array of OWSCs the near-resonant mechanisms shown in figure 6 were never described before. Since for an OWSC the exciting torque $F^{\prime}$ is the power-generating action [19], the near-resonant behaviour of $F^{\prime}$ (see again figure 6) suggests potential for constructive interaction in a finite array, which is now investigated. Generally, given a wave farm of $M$ identical WECs, an interaction factor can be defined as the ratio between the 
total power $P^{\prime}$ extracted by the system and $M$ times the power $P_{s}^{\prime}$ generated by a single device in the open ocean, i.e.

$$
q=\frac{P^{\prime}}{M P_{s}^{\prime}} .
$$

A value of $q<1$ indicates that the array interactions have an overall negative impact on the absorbing power of the farm and corresponds to destructive interference; conversely $q>1$ corresponds to constructive interference. Noting that $q$ (42) hides the real amount of absorbed power by each device, Babarit [8] introduced a modified version of the interaction factor for an array of $M$ identical elements:

$$
q_{m}^{m o d}=\frac{P_{m}^{\prime}-P_{s}^{\prime}}{\max _{T} P_{s}^{\prime}}, \quad m=1, \ldots, M,
$$

where $P_{m}^{\prime}$ is the power output by the $m$ th device and the maximum must be taken over a selected period interval (e.g. $5-15 \mathrm{~s}$ in this paper). When $q_{m}^{\text {mod }}>0$, interference effects increase the absorbed power by the $m$ th element with respect to the isolated case. Figure 7(a) shows the behaviour of the interaction factor $q$ (42) versus the period of the incident wave for the two-flap system, the three-flap system and the infinite array described above. As expected $[3,5,8]$, the $q$ factor shows regions of constructive $(q>1)$ and destructive $(q<1)$ interaction. Comparison of figure 6 and figure 7 (a) shows that the maximum constructive interaction, corresponding to the largest $q$ factor, is attained in all the systems when the relevant exciting torque is maximum, i.e. at near resonance. This implies that near-resonant interactions arising in a finite in-line array are beneficial to the efficiency of the system, when the flap width is comparable to the gap. In figure 7(b) the modified interaction factor (43) is plotted against the period of the incident waves, again for either flap of a two-flap system, the central flap of a threeflap system and any flap of an infinite array. Note from figure 7 that regions where $q>1$ correspond to $q_{m}^{\text {mod }}>0$. This means that for a wave farm of in-line OWSCs, constructive interference $(q>1)$ is usually accompanied by an actual increase of absorbed power by the single elements $\left(q_{m}^{\text {mod }}>0\right)$. This is a peculiar property of OWSCs and does not yield in general for systems of different converters (e.g. heaving or surging buoys, see [8]), confirming that the interference effects activating in a wave farm of in-line OWSCs at near resonance are actually beneficial to wave power extraction. 
(a)

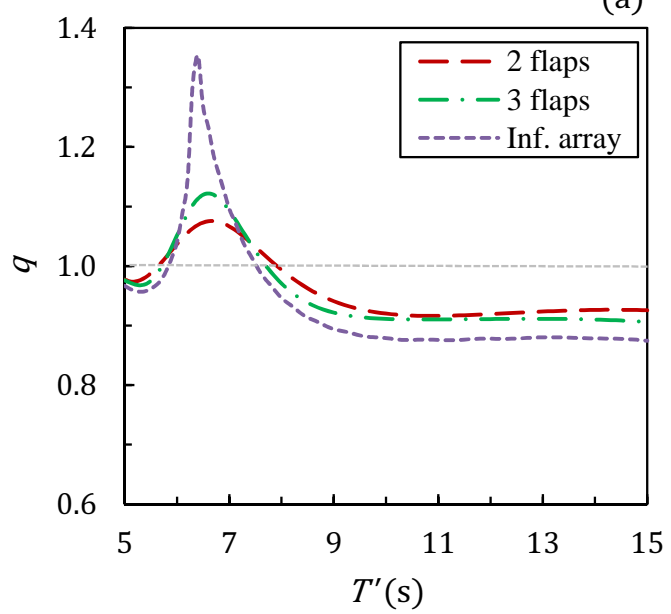

(b)

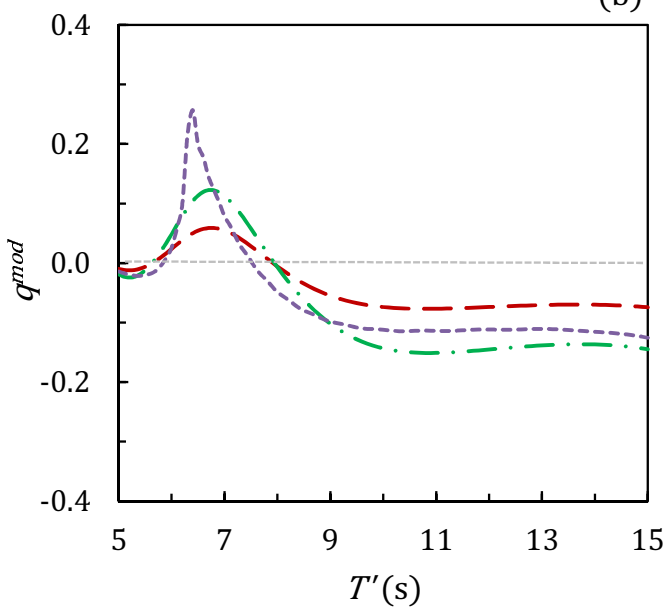

Figure 7: (a) Interaction factor (42) versus period of the incident wave for a two-flap system (dashed line), a three-flap system (dash-dotted line) and an infinite array of flaps oscillating at unison (dotted line). (b) Modified interaction factor $q_{m}^{\bmod }$ for either flap of a two-flap system $\left(q_{1}^{\text {mod }}=q_{2}^{\text {mod }}\right.$, dashed line $)$, the central flap of a three-flap system $\left(q_{2}^{\text {mod }}\right.$, dash-dotted line) and any flap of an infinite array (dotted line). The geometry is the same as in figure 6. Values are calculated with the semi-analytical model of $\S 2.3$ for the finite arrays and with the semi-analytical model of Renzi \& Dias [20] for the infinite array.

\subsubsection{Influence of flap spacing}

In this section we investigate the parametric dependence of an in-line array of OWSCs on the flap spacing, with the semi-analytical model of $\S 2.3$. A system of two in-line flaps is considered, with $w_{1}^{\prime}=w_{2}^{\prime}=26 \mathrm{~m}, c_{1}^{\prime}=c_{2}^{\prime}=$ $4 \mathrm{~m}$ and $h^{\prime}=13 \mathrm{~m}$. Waves of amplitude $A_{I}^{\prime}=1 \mathrm{~m}$ are normally incident on the flaps. Figure 8 shows the behaviour of the $q$ factor versus the gap width $s^{\prime}$ for the two-flap system, respectively for $T^{\prime}=7 \mathrm{~s}$ and $T^{\prime}=10 \mathrm{~s}$. Not surprisingly (see [3]), the plots of figure 8 show a sequence of constructive and destructive interference regions. Note also that the interference effects get weaker as the gap width $s^{\prime}$ becomes nearly three times larger than the flap width, i.e. $q \simeq 1$ as $s^{\prime} \gtrsim 3 w^{\prime}$. Given the period $T^{\prime}$ of the incident wave, figure 8 also shows that it is possible to identify an optimum gap width $s_{\text {opt }}^{\prime}\left(T^{\prime}\right)$ corresponding to the maximum interaction factor, i.e. to the strongest constructive interference. The optimum gap width for the two-flap array under analysis is reported in figure $8(\mathrm{~b})$ together with the optimum gap width for an infinite array made by the same flaps, for both periods of the incident wave [20]. Note that in both cases $s_{\text {opt }}^{\prime}$ for the 2-flap array is smaller than $s_{\text {opt }}^{\prime}$ for the infinite array. This is clearly a consequence of the near- 
(a)

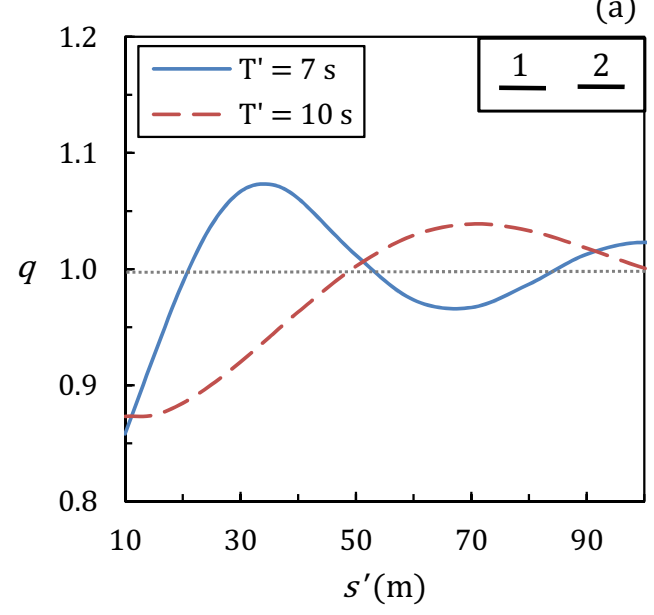

(b)

\begin{tabular}{lcc}
\hline Configuration & $s^{\prime}{ }_{\text {opt }}(7 \mathrm{~s})$ & $s^{\prime}{ }_{\text {opt }}(10 \mathrm{~s})$ \\
\hline 2 flaps & 35 & 70 \\
\hline Infinite array & 39 & 77 \\
\hline
\end{tabular}

Figure 8: (a) Interaction factor (42) versus gap width $s^{\prime}$ for a two-flap system and two different periods of the incident wave, calculated with the semi-analytical model. The system configuration is sketched at the top right corner (not in scale). Parameters are: $w_{1}^{\prime}=w_{2}^{\prime}=26 \mathrm{~m}, c_{1}^{\prime}=c_{2}^{\prime}=4 \mathrm{~m}$ and $h^{\prime}=13 \mathrm{~m}$. Waves of amplitude $A_{I}^{\prime}=1 \mathrm{~m}$ are normally incident on the system. (b) Values (in metres) of the optimum gap width for both periods of the incident wave, for a system of 2 in-line flaps and an infinite array. The latter are calculated with the model of Renzi \& Dias [20].

resonant phenomenon discussed in $§ 3.2 .1$. Since the interactions in a finite array are weaker than in an infinite array, in the finite array configuration the flaps need to be closer than they would need to be in the infinite array in order to interfere constructively. Such considerations should influence the designer's choice on the number of flaps and the optimal flap spacing during the design process of a wave farm of OWSCs. In the following section, we shall apply the numerical model of $\S 2.4$ to analyse a configuration of practical engineering interest which cannot be studied with the analytical approach.

\section{Application}

Here we apply the numerical model of $\S 2.4$ to investigate a staggered array configuration, resembling the one envisaged by APL for a wave farm of Oyster WECs [41]. Figure 9 shows a sketch of the layout, where the two OWSCs have the same dimensions as those of $\S 3$ and the waves are normally incident. The PTO coefficient is again the same for both converters, i.e. $\eta_{1}^{\prime}=\eta_{2}^{\prime}$ and since the analytical approach cannot be used to optimise the PTO for such configuration - has been tuned in order to achieve the optimum power of an isolated converter (see also [8] for a similar technique). However, this does 


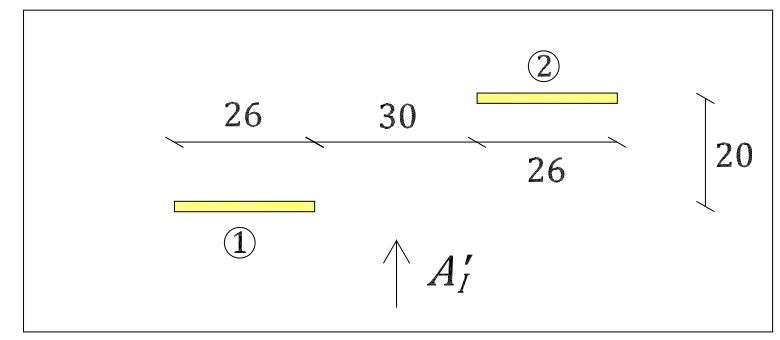

Figure 9: Geometry of an array made by 2 staggered OWSCs in normally incident waves. Values are in metres.

not imply loss of physical insight, since in this case we are mainly concerned about investigating the variation of the wave field and the hydrodynamic actions on the flaps induced by the loss of symmetry in the system. Figure 10 shows a snapshot of the free-surface elevation calculated with the numerical model for an array of two in-line (left panel) and two staggered (right panel) flaps, held fixed in incoming waves (scattering problem). When a crest is about to hit the array in the in-line system, a large difference in free-surface elevation (and hence in pressure) generates between the sides of the flaps, as shown in figure 10 (left panel). Here, if allowed to move both flaps would pitch towards the right pushed by a clockwise net torque. On the contrary, in a staggered array spatial separation along the direction of propagation of the waves makes it impossible for a single wave front to impinge on both flaps at the same time. As a consequence, the flaps are likely to experience different pressure jumps between their sides. This is clear in figure 10 (right panel), where the first flap undergoes a jump in pressure between its sides, while the second flap is all surrounded by a trough, which yields little pressure difference. If allowed to move, the front flap (1) would now pitch towards the right while the back flap (2) would remain nearly motionless. This new dynamics has clear effects on the exciting torque curves. Figure 11(a) shows the plot of the exciting torque versus the period of the incident waves for both flaps in the staggered array. Due to the loss of symmetry, the variation of the exciting torque with respect to the wave period is less predictable than before. Note that a similar general result has also been obtained by Chatjigeorgiou [42] for a system of two staggered truncated elliptical cylinders, when moving from a symmetric to a non-symmetric configuration. For the staggered OWSCs of figure 9, the curve of the front flap peaks at a larger 

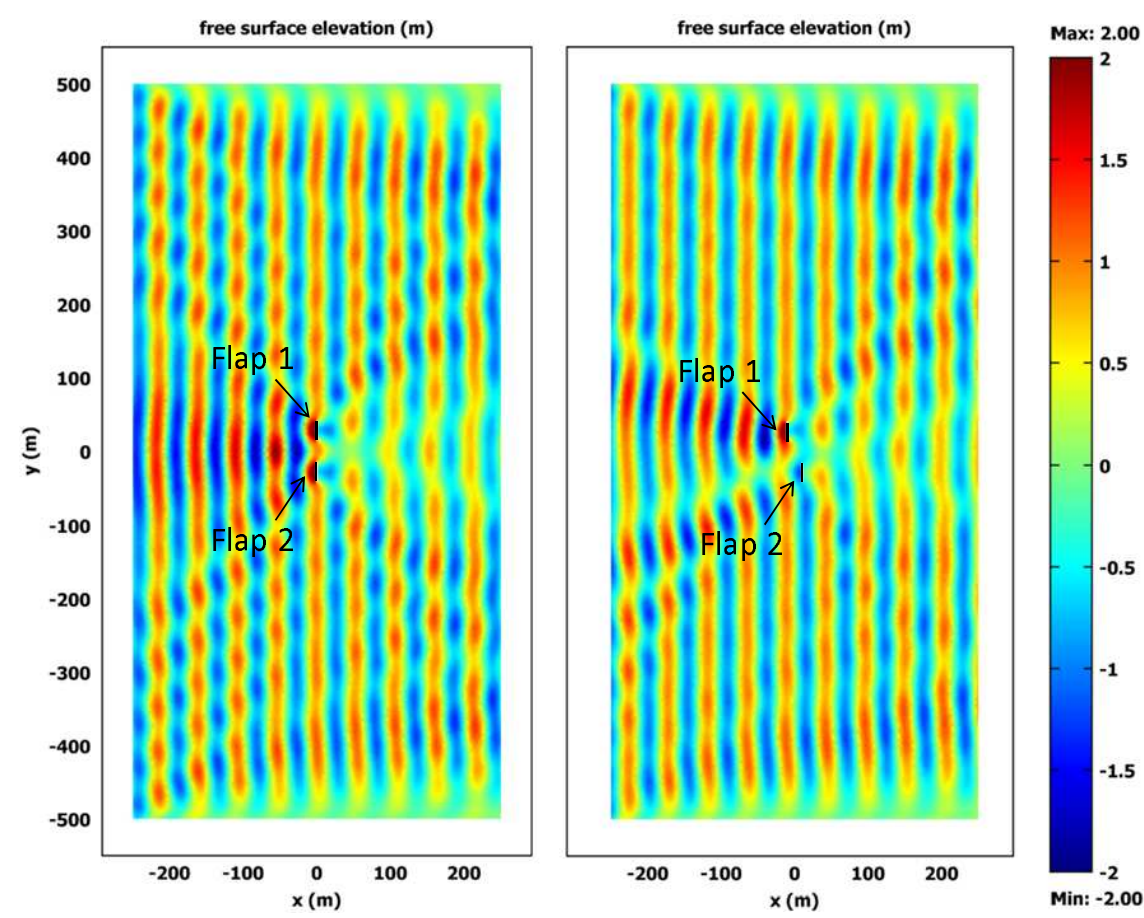

Figure 10: Snapshot of the free-surface elevation for two flaps in (a) an in-line configuration, (b) the staggered configuration of figure 9. Both flaps are held fixed in incoming waves (scattering problem). In the plot, the thickness of the flaps has been exaggerated for easiness of reading. Waves of amplitude $A_{I}^{\prime}=1 \mathrm{~m}$ and period $T^{\prime}=6 \mathrm{~s}$ are normally incident on the system from the left. Values are calculated with the numerical model of $\S 2.4$. 

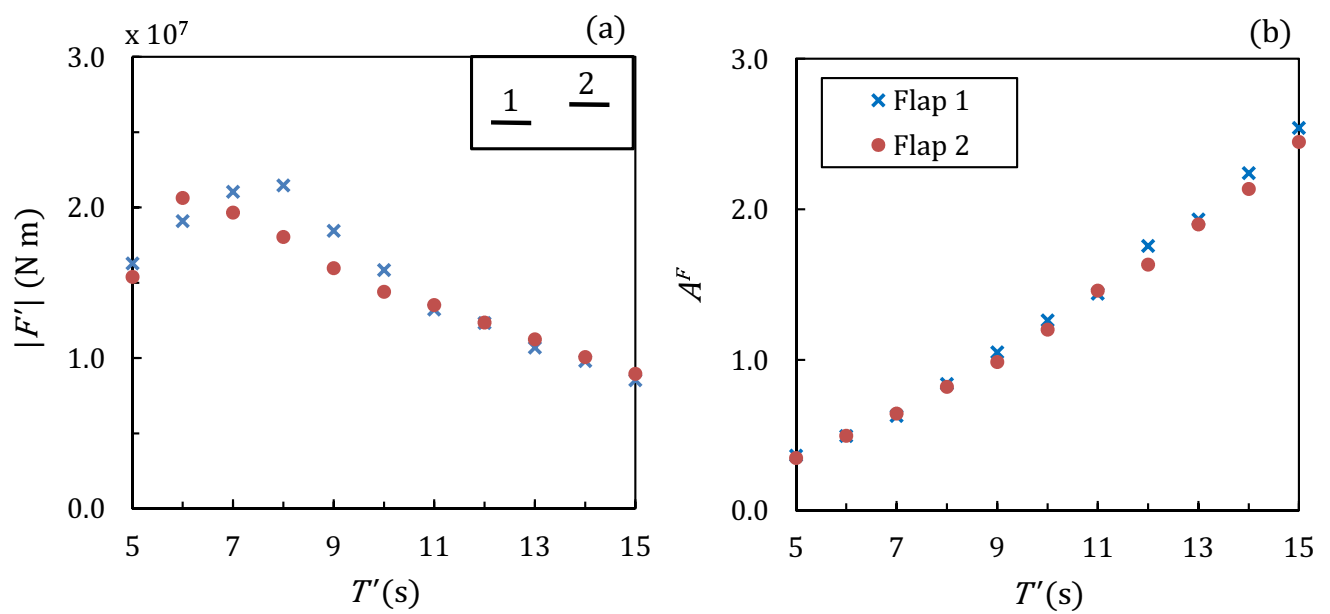

Figure 11: (a) Exciting torque and (b) amplitude factor versus period of the incident waves for the staggered array of figure 9 . The system configuration is sketched at the top right corner of (a) (not in scale). Waves of amplitude $A_{I}^{\prime}=1 \mathrm{~m}$ are normally incident on the system. Values are calculated with the numerical model of $\S 2.4$.

period than that of the back flap (see again figure 11a). This determines a reduction in synergy between the two OWSCs, each now working better at a different period than the other. This in turn rules out the concurrent occurrence of efficiency-enhancing near-resonant interactions at the same period for both flaps. Overall, those dynamics are expected to be detrimental for the efficiency of the farm. In order to quantify the reduction in efficiency, the amplification factors of both OWSCs are plotted in figure 11(b) against the period of the incident waves. Even though a certain loss of efficiency was predictable, comparison of figure 11(b) with figure 4(b) shows quite surprisingly that the amplification factors of the staggered system are almost halved with respect to the relevant values of the in-line system. Indeed such a large loss of efficiency is partially due to the usage of non-optimal PTO damping in the staggered configuration. Also, other parameters like the longitudinal and latitudinal spacing between the flaps could be varied, making it possible to increase the wave power output. Such analysis is not undertaken here and could be the topic of a different research project.

\section{Conclusions}

A three-dimensional potential flow theory has been developed for the hydrodynamic analysis of a finite array of OWSCs. A semi-analytical and a 
fully numerical approach have been considered in order to solve the governing equations of the system. Results of both models for systems of two and three in-line converters resembling the Oyster $800^{\mathrm{TM}}$ OWSC developed by Aquamarine Power Limited are compared, showing good agreement between the two approaches. Parametric analysis is then undertaken with the semianalytical model in order to gain a further insight on the system dynamics and investigate its dependence on the system main parameters. While investigating the influence of the number of flaps on the array performance, a near-resonant phenomenon is identified. Because of near resonance, peaks in the exciting torque acting on the elements of a finite array manifest at periods close to the transverse-mode resonance of an infinite array [20]. Such near-resonant mechanism is responsible for the occurrence of constructive interference in the array (i.e. $q>1$ ), which is accompanied by an effective increase of absorbed power by the single elements (i.e. $q_{m}^{\bmod }>0$ ). This is a distinctive property of an array of OWSCs and does not yield in general for systems of different converters, like heaving or surging buoys, suggesting that the near-resonant effects activating in a wave farm of in-line OWSCs are actually beneficial to wave power extraction. Parametric analysis for an in-line array also shows that, given the period of the incident wave, there exists an optimum gap width for which the constructive interaction among the flaps is maximum. Such result could be used as a preliminary design criterion for an array of OWSCs. The numerical model is then applied to analyse a configuration of practical engineering interest, i.e. an array of two staggered flaps, which cannot be studied with the analytical approach of 2.3. The case analysed here shows that loss of symmetry in the system can be detrimental to the array efficiency. Such mechanism could be partially prevented by using an appropriate control of the PTO system and an optimisation of the geometric layout.

The analysis performed in this paper assumes monochromatic incident waves. In real seas, the presence of several spectral wave components would certainly modify the system behaviour. Nevertheless, the fundamental dynamics would be still governed by the mechanisms discussed in this paper. In particular, in random seas the near-resonant behaviour of the array could be exploited by designing the flap spacing so that the resulting $q$-factor curve couples well with the wave spectrum of the incident sea. However, we expect wave interaction effects to be weaker in random waves than in monochromatic waves, due to partial compensation of constructive and destructive effects over the whole spectrum (see for example [8]). Finally, a separate 
analysis must be carried out in order to investigate the existence of motionresonant modes for an in-line array of OWSCs. Motion resonance is a radiation phenomenon and is different from the near resonance described here, which is instead a scattering phenomenon. Motion resonance could occur for an articulated system of flaps at certain periods of the incident wave, for which the angular oscillations are maximised (perfect trapping for a system of flood gates in a channel has been described for the first time by Mei et al. [25]). Investigation is currently ongoing to determine whether motionresonant modes can occur for a system of OWSCs and will be disclosed in the near future.

The work of E.R. and F.D. was funded by Science Foundation Ireland (SFI) under the research project "High-end computational modelling for wave energy systems". A.A. and G.B. would like to acknowledge the research projects FP7-OCEAN.2011-1 "MERMAID: Innovative Multi-purpose offshore platforms: planning, design and operation" and FP7-PEOPLE-2009IRSES "Sim.COAST: Numerical Simulation Tools for Protection of Coasts against Flooding and Erosion". Fruitful discussions with the Research and Development team of Aquamarine Power Limited are kindly acknowledged.

\section{Appendix A. Semi-analytical solution}

In this section the system of equations (28)-(29) for the $n$ th-mode $2 \mathrm{D}$ spatial potentials $\varphi_{n}^{D}$ and $\varphi_{n}^{\alpha}$ (24) will be solved by extending the semianalytical approach of Renzi \& Dias [22] to a finite system of flaps. First consider the 2D Green function

$$
G_{n}(x, y ; \xi, \eta)=\frac{1}{4 i} H_{0}^{(1)}\left(\kappa_{n} \rho\right),
$$

solution of the system

$$
\left(\nabla^{2}+\kappa_{n}^{2}\right) G_{n}=0, \quad G_{n} \rightarrow \frac{\ln \rho}{2 \pi} \text { as } \rho \rightarrow 0,
$$

where $\rho=\sqrt{(x-\xi)^{2}+(y-\eta)^{2}},(\xi, \eta) \in \mathbb{R}^{2}[19]$. In (A.1), $H_{0}^{(1)}$ is the Hankel function of the first kind and 0th order, outgoing for large argument, while $\kappa_{0}=k$ and the $\kappa_{n}=i k_{n}$ are still the solutions of the dispersion relation (26) for $n>0$. Application of Green's integral theorem [12] to a 2D surface 
enclosed by a large circumference centred at the origin $O$ and containing all flaps yields

$$
\left\{\begin{array}{l}
\varphi^{D}(x, y) \\
\varphi_{n}^{\alpha}(x, y)
\end{array}\right\}=\left.\sum_{m=1}^{M} \int_{y_{m}^{s}}^{y_{m}^{e}}\left\{\begin{array}{c}
\Delta \varphi_{n m}^{D}(\eta) \\
\Delta \varphi_{n m}^{\alpha}(\eta)
\end{array}\right\} G_{n, \xi}(x, y ; \xi, \eta)\right|_{\xi=0} d \eta
$$

for either potential, where the notation is that of $\S 2.3$. In (A.2), the subscript after the vertical bar indicates the point at which the derivative must be calculated and

$$
\left\{\begin{array}{c}
\Delta \varphi_{n m}^{D}(y) \\
\Delta \varphi_{n m}^{\alpha}(y)
\end{array}\right\}=\left\{\begin{array}{c}
\varphi_{n}^{D}\left(0^{-}, y\right) \\
\varphi_{n}^{\alpha}\left(0^{-}, y\right)
\end{array}\right\}-\left\{\begin{array}{c}
\varphi_{n}^{D}\left(0^{+}, y\right) \\
\varphi_{n}^{\alpha}\left(0^{+}, y\right)
\end{array}\right\}, y \in\left(y_{m}^{s}, y_{m}^{e}\right) .
$$

The first line of (A.3) indicates the jump in the $n$th diffraction potential across the $m$ th flap, when all the flaps are fixed. Similarly, the second line of (A.3) indicates the jump in the $n$-th radiation potential across the $m$ th flap when the $\alpha$ th flap is moving and the remaining flaps are fixed. Note that if $M=1$, (A.2) reduces to expression (A.1) of Renzi \& Dias [22] for a single flap in the open ocean. On the other hand, if $M=\infty$ and assuming that all flaps move at unison, (A.2) reduces to expression (B7) of Renzi \& Dias [19] for a flap in a channel, equivalent to an infinite array of flaps oscillating at unison in the open ocean [20]. Back to the finite array, now focus on one flap at a time, say the $\beta$ th flap. Application of (29) to the $\beta$ th flap together with the integral form (A.2) and (A.1) yields the following hypersingular integral equations for the jumps in potential across such flap:

$$
\begin{array}{r}
\sum_{m=1}^{M} H \cdot \int_{y_{m}^{s}}^{y_{m}^{e}}\left\{\begin{array}{c}
\Delta \varphi_{n m}^{D} \\
\Delta \varphi_{n m}^{\alpha}
\end{array}\right\}(\eta) \frac{H_{1}^{(1)}\left(\kappa_{n}|y-\eta|\right)}{|y-\eta|} d \eta= \\
\frac{4 i}{\kappa_{n}}\left\{\begin{array}{c}
A_{I} d_{n} \\
f_{n \alpha} \delta_{\alpha \beta}
\end{array}\right\}, \quad y \in\left(y_{\beta}^{s}, y_{\beta}^{e}\right) .
\end{array}
$$

The latter must then be applied in succession to all flaps, $\beta=1, \ldots, M$ to ensure that the solution fully satisfies the b.c.'s (29). In (A.4), the symbol $H$. before the integral sign indicates a Hadamard finite-part integral [19, 20, 22], whose kernel is singular at $\eta=y$. In order to resolve this singularity, perform the following change of variables inside the integral of (A.4):

$$
u_{m}=y_{m}^{s}+y_{m}^{e}, \zeta=\frac{2 \eta-u_{m}}{w_{m}},\left\{\begin{array}{c}
\Delta \varphi_{n m}^{D}(\eta) \\
\Delta \varphi_{n m}^{\alpha}(\eta)
\end{array}\right\}=\left\{\begin{array}{c}
P_{n m}(\zeta) \\
Q_{n \alpha m}(\zeta)
\end{array}\right\}
$$


with $\zeta \in(-1,1)$ and define

$$
v_{m}(y)=\frac{2 y-u_{m}}{w_{m}}=\frac{2 y-y_{m}^{s}-y_{m}^{e}}{y_{m}^{e}-y_{m}^{s}}, \quad y \in\left(y_{\beta}^{s}, y_{\beta}^{e}\right),
$$

where $m$ indicates any flap and $\beta$ refers to the flap on which the b.c. (A.4) is being applied in succession. In (A.5) $P_{n m}$ indicates the jump in the $n$th $2 \mathrm{D}$ diffraction potential across the generic $m$ th flap in the new system of variables. Similarly, $Q_{\text {nam }}$ denotes the jump in the $n$th 2 D radiation potential across the $m$ th flap when the $\alpha$ th flap is moving and all the other flaps are fixed. Now let $m=\gamma$ denote any but the $\beta$ th flap, i.e. $\gamma \neq \beta$. Hence substituting (A.5) into the boundary condition (A.4) on flap $\beta$ and separating the term $m=\beta$ in the summation (A.4) from all other terms $m=\gamma \neq \beta$ yields

$$
\begin{array}{r}
H \cdot \int_{-1}^{1}\left\{\begin{array}{c}
P_{n \beta}(\zeta) \\
Q_{n \alpha \beta}(\zeta)
\end{array}\right\} \frac{H_{1}^{(1)}\left(\kappa_{n} \frac{w_{\beta}}{2}\left|v_{\beta}(y)-\zeta\right|\right)}{\left|v_{\beta}(y)-\zeta\right|} d \zeta \\
+\sum_{\gamma=1}^{M} \int_{-1}^{1}\left\{\begin{array}{c}
P_{n \gamma}(\zeta) \\
Q_{n \alpha \gamma}(\zeta)
\end{array}\right\} \frac{H_{1}^{(1)}\left(\kappa_{n} \frac{w_{\gamma}}{2}\left|v_{\gamma}(y)-\zeta\right|\right)}{\left|v_{\gamma}(y)-\zeta\right|} d \zeta \\
=\frac{4 i}{\kappa_{n}}\left\{\begin{array}{c}
A_{I} d_{n} \\
f_{n \alpha} \delta_{\alpha \beta}
\end{array}\right\}, \quad y \in\left(y_{\beta}^{s}, y_{\beta}^{e}\right),
\end{array}
$$

where $\sum^{\prime}$ indicates that the sum must be evaluated for all $\gamma \neq \beta$. This separation allows to assess the singular behaviour of the kernels in (A.7). For $m=\beta$, (A.6) reveals that $v_{\beta}(y) \in(-1,1)$. Hence the kernel of the first integral in (A.7) is singular when $\zeta=v_{\beta}(y) \in(-1,1)$. On the other hand, for $m=\gamma \neq \beta$, (A.6) reveals that $v_{\gamma}(y) \notin(-1,1)$, so that $\zeta \neq v_{\gamma}(y)$ and the kernels of the integrals inside the summation of (A.7) are never singular. Hence the singularity is now confined in the first Hadamard finitepart integral of (A.7). The latter can be finally solved by following the general method devised by Renzi \& Dias [19]. First expand the Hankel function inside the first integral of (A.7) according to $\$ 8.444$ of Gradshteyn \& Ryzhik [43] as

$$
H_{1}^{(1)}\left(\kappa_{n} \frac{w_{\beta}}{2}\left|v_{\beta}(y)-\zeta\right|\right)=\frac{4}{i \pi} \frac{1}{\kappa_{n} w_{\beta}\left|v_{\beta}(y)-\zeta\right|}+R_{n}\left(\kappa_{n} \frac{w_{\beta}}{2}\left|v_{\beta}(y)-\zeta\right|\right),
$$


where

$$
\begin{array}{r}
R_{n}(z)=J_{1}(z)\left[1+\frac{2 i}{\pi}\left(\ln \frac{z}{2}+\sigma\right)\right] \\
-\frac{i}{\pi}\left[\frac{z}{2}+\sum_{j=2}^{+\infty} \frac{(-1)^{j+1}(z / 2)^{2 j-1}}{j !(j-1) !}\left(\frac{1}{j}+\sum_{q=1}^{j-1} \frac{2}{q}\right)\right],
\end{array}
$$

$J_{1}$ is the Bessel function of first kind and first order and $\sigma=0.577215 \ldots$ the Euler constant. Then also expand the unknown jumps in potential as

$$
\left\{\begin{array}{c}
P_{n m}(\zeta) \\
Q_{n \alpha m}(\zeta)
\end{array}\right\}=\left(1-\zeta^{2}\right)^{1 / 2} \sum_{p=0}^{\infty}\left\{\begin{array}{c}
A_{I} a_{n m p} \\
b_{n \alpha m p}
\end{array}\right\} U_{p}(\zeta), \quad m=1, \ldots, M
$$

where $U_{p}$ is the Chebyshev polynomial of the second kind and order $p \in \mathbb{N}$, while the $a_{n m p}$ and $b_{n \alpha m p}$ are unknown complex coefficients to be determined. Substituting (A.8) and the series expansions (A.9) into the hypersingular integral equations (A.7) for each $\beta=1, \ldots, M$ in succession and developing some lengthy algebra [see 19, for a similar procedure] finally yields

$$
\begin{array}{r}
\sum_{p=0}^{\infty}\left\{\left\{\begin{array}{c}
a_{n \beta p} \\
b_{n \alpha \beta p}
\end{array}\right\} C_{n \beta p}\left(v_{\beta}\right)+\sum_{\gamma=1}^{M},\left\{\begin{array}{c}
a_{n \gamma p} \\
b_{n \alpha \gamma p}
\end{array}\right\} D_{n \beta \gamma p}\left(v_{\beta}\right)\right\} \\
=-\pi w_{\beta}\left\{\begin{array}{c}
d_{n} \\
f_{n \alpha} \delta_{\alpha \beta}
\end{array}\right\}, \quad v_{\beta} \in(-1,1), \beta=1, \ldots, M
\end{array}
$$

57

where the orthogonality property of the Chebyshev polynomials has also been used [see 19]. In (A.10)

$$
\begin{aligned}
C_{n \beta p}\left(v_{\beta}\right) & =-\pi(p+1) U_{p}\left(v_{\beta}\right)+\frac{i \pi \kappa_{n} w_{\beta}}{4} \\
& \times \int_{-1}^{1} \frac{\left(1-\zeta^{2}\right)^{1 / 2} U_{p}(\zeta) R_{n}\left(\frac{1}{2} \kappa_{n} w_{\beta}\left|v_{\beta}-\zeta\right|\right)}{\left|v_{\beta}-\zeta\right|} d \zeta
\end{aligned}
$$

and

$$
\begin{aligned}
D_{n \beta \gamma p}\left(v_{\beta}\right) & =\frac{i \pi \kappa_{n} w_{\beta} w_{\gamma}}{4} \int_{-1}^{1}\left(1-\zeta^{2}\right)^{1 / 2} U_{p}(\zeta) \\
& \times \frac{H_{1}^{(1)}\left(\frac{1}{2} \kappa_{n} \mid v_{\beta} w_{\beta}+u_{\beta}-u_{\gamma}-w_{\gamma} \zeta\right)}{\left|v_{\beta} w_{\beta}+u_{\beta}-u_{\gamma}-w_{\gamma} \zeta\right|} d \zeta
\end{aligned}
$$


are complex coefficients which can be evaluated numerically. Expression (A.10) defines two different systems of linear equations for given $n$ and $\alpha$, both valid for any $v_{\beta} \in(-1,1)$. The unknowns are the complex coefficients $a_{n m p}$ and $b_{n \alpha m p}$ of the series expansions (A.9). Note that in (A.10) the term $C_{n \beta p}$ indicates the $n$th mode, $p$ th order contribution of the $\beta$ th-flap on itself, while $D_{n \beta \gamma p}$ indicates the $n$th mode, $p$ th order contribution of the $\gamma$ th-flap on the $\beta$ th-flap. As discussed by Renzi \& Dias [19, 20, 22], systems like (A.10) can be solved numerically with a collocation scheme by truncating the external summation to a finite integer $p=P$ and evaluating the coefficients (A.11) and (A.12) at the specific points

$$
v_{\beta}=v_{q}=\frac{\cos (2 q+1) \pi}{2 P+2}, \quad q=0,1, \ldots, P,
$$

for any $\beta$, which correspond to the zeros of the Chebyshev polynomials of the first kind. As a consequence, (A.10) transforms into

$$
\begin{aligned}
& \sum_{p=0}^{P}\left\{\left\{\begin{array}{c}
a_{n \beta p} \\
b_{n \alpha \beta p}
\end{array}\right\} C_{n \beta p}\left(v_{q}\right)+\sum_{\gamma=1}^{M},\left\{\begin{array}{c}
a_{n \gamma p} \\
b_{n \alpha \gamma p}
\end{array}\right\} D_{n \beta \gamma p}\left(v_{q}\right)\right\} \\
& =-\pi w_{\beta}\left\{\begin{array}{c}
d_{n} \\
f_{n \alpha} \delta_{\alpha \beta},
\end{array}\right\}, \quad q=0, \ldots, P ; \beta=1, \ldots, M .
\end{aligned}
$$

The first system (upper line of A.13) yields the series coefficients $a_{n m p}$ for the jump in potential of the diffraction problem $P_{n m}$ (A.9), for each modal order $n$ and body $m$. The second system (lower line of A.13) yields the series coefficients $b_{n \alpha m p}$ for the jump in potential of the radiation problem $Q_{n \alpha m}$ (A.9) for each mode $n$ and body $m$, when the $\alpha$ th-body is moving and the others are at rest. Now note from (30) that $d_{n}=0$ for $n>0$. As a consequence, the diffraction system (upper line of A.13) becomes the homogeneous form of the radiation system (lower line of A.13) for $n>0$. Hence in order the solution of the radiation problem to be unique, it must be $a_{n \beta p}=0, n>0$. Now, the vertical eigenmodes with $n>0$ correspond to imaginary solutions of the dispersion relation $\omega^{2}=k \tanh k h$, i.e. such terms represent evanescent modes [12]. Hence the fact that $a_{n \beta p}=0$ for $n>0$ physically implies that the incident wave is not able to excite evanescent modes when interacting with the system of in-line fixed plates [see also 19, 20, 22, for a similar behaviour].

Now given the most generic configuration with $M$ bodies and considering $N$ vertical eigenmodes in the radiation problem, the above method requires 
the solution of $M \times(P+1)$ linear equations for the diffraction problem and of $N \times M \times M \times(P+1)$ linear equations for the radiation problem. However, occurrence of symmetries in the geometric layout of the system can strongly simplify (A.13) and reduce the number of unknowns (this is the case, for example, of a series of in-line identical flaps). Incidentally, consider the case when there is only one body of width $w$, i.e. $M=1$. Here there are no mutual contributions and the $D_{n \beta \gamma p}=0$ in (A.13). As a consequence, the latter strongly simplifies to

$$
\sum_{p=0}^{\infty}\left\{\begin{array}{c}
a_{n 1 p} \\
b_{n 11 p}
\end{array}\right\} C_{n 1 p}\left(v_{q}\right)=-\pi w\left\{\begin{array}{c}
d_{n} \\
f_{n 1},
\end{array}\right\}, \quad q=0, \ldots, P
$$

which corresponds exactly to the system (A.9) of Renzi \& Dias [22] for a single flap in the open ocean. For a general number of flaps, (A.13) gives the complex coefficients of the series expansions in (A.9), from which the jumps in diffraction and radiation potentials can be calculated according to (A.5). Substitution of the jumps in potential into Green's formula (A.2) and then into the decomposition (24) finally yields the sought complex spatial potentials $\phi^{D}(32)$ and $\phi^{(\alpha)}(33)$.

\section{Appendix B. Reciprocity relation}

Consider the complex $2 \mathrm{D}$ radiation potentials $\varphi_{n}^{\alpha_{1}}$ and $\varphi_{n}^{\alpha_{2}}$, with $\alpha_{1} \neq \alpha_{2}$. As shown in $\S 2.3$, both potentials must satisfy the Helmholtz equation

$$
\left(\nabla^{2}+\kappa_{n}^{2}\right)\left\{\begin{array}{c}
\varphi_{n}^{\alpha_{1}} \\
\varphi_{n}^{\alpha_{2}}
\end{array}\right\}=0
$$

the kinematic boundary conditions

$$
\left\{\begin{array}{l}
\varphi_{n, x}^{\alpha_{1}} \\
\varphi_{n, x}^{\alpha_{2}}
\end{array}\right\}=\left\{\begin{array}{l}
f_{n \alpha_{1}} \delta_{\alpha_{1} m} \\
f_{n \alpha_{2}} \delta_{\alpha_{2} m}
\end{array}\right\} \quad \text { on } \partial \mathcal{L}_{m}, m=1, \ldots, M
$$

and be outgoing at large distance from the array. Application of Green's integral theorem in a large circular region enclosing all the flaps to $\varphi_{n}^{\alpha_{1}}$ and $\varphi_{n}^{\alpha_{2}}$ together with (B.1)-(B.2) yields the relation

$$
f_{n \alpha_{2}} \int_{y_{\alpha_{2}}^{s}}^{y_{\alpha_{2}}^{e}} \Delta \varphi_{n \alpha_{2}}^{\alpha_{1}}(y) d y=f_{n \alpha_{1}} \int_{y_{\alpha_{1}}^{s}}^{y_{\alpha_{1}}^{e}} \Delta \varphi_{n \alpha_{1}}^{\alpha_{2}}(y) d y
$$


Substituting the change of variables (A.5) and the series expansion (A.9) into (B.3), renaming $\alpha_{1}=\alpha$ and $\alpha_{2}=\beta$ and developing the algebra finally gives

$$
w_{\beta} f_{n \beta} b_{n \alpha \beta 0}=w_{\alpha} f_{n \alpha} b_{n \beta \alpha 0},
$$

which is a reciprocity relation between the 0th-order coefficients of the Chebyshev expansion (A.9) for the radiation problem.

\section{Appendix C. Numerical procedure}

Since the mathematical problem is hyperbolic, it is solved by means of a time-marching numerical scheme. In order to correctly reproduce the wave field, the minimum mesh element size must be at least one tenth of the simulated wave length. It is important for the efficiency of the timestepping algorithm to assemble the time independent matrices only once. The Generalized- $\alpha$ method is used. This is a one-step implicit method for solving the transient problem, which attempts to increase the amount of numerical damping present without degrading the order of accuracy. Such method allows for high frequency energy dissipation and second order accuracy. The method was first developed for the second-order equations in structural mechanics $[44,45]$. This linear system solver uses the restarted generalized minimum residual (GMRES) with incomplete LU preconditioner for fast convergence. The latter is an iterative method for general linear systems of the form $A x=b$. For models with many degrees of freedom (roughly, more than 100,000 to 1,000,000 depending on available computer memory), the direct solvers typically require too much memory. In those cases, the more memory-efficient iterative solvers like GMRES perform better. However, iterative solvers are less stable than direct solvers in that they do not always converge (i.e. arrive at a solution). For the simulations carried out in this paper, the value of the number of iterations is 50. A larger restart value increases the robustness of the interactive procedure, but it also increases memory use and computational time. For large problems, the computational cost is often very large to produce a preconditioner of such a high quality that the termination criteria are fulfilled for a small number of iterations and for a small restart value. For those problems it is often advantageous to set up a preconditioner with a somewhat lesser quality and instead increase the restart value or iterate more steps. Doing so typically increases the condition number for the preconditioned system, so an increase in the error-estimate 
factor might be needed as well. The incomplete LU preconditioner performs an incomplete LU factorization of the system matrix $A$. That is, it drops small elements during the column-oriented Gaussian elimination. Thus it saves memory, and the resulting factors $\mathrm{L}$ and $\mathrm{U}$ are approximate. The numerical solution is obtained using the software COMSOL Multiphysics.

\section{References}

[1] Budal, K. 1977. Theory for absorption of wave power by a system of interacting bodies. Journal of Ship Research, 21(4), 248-253.

[2] Srokosz, M.A. \& Evans, D.V. 1979. A theory for wave-power absorption by two independently oscillating bodies. Journal of Fluid Mechanics, 90(2), 337-362.

[3] Falnes, J. 1980. Radiation impedance matrix and optimum power absorption for interacting oscillators in surface waves. Applied Ocean Research, 2(2), 75-80.

[4] Thomas, G.P. and Evans, D.V. 1981. Arrays of three-dimensional waveenergy absorbers. Journal of Fluid Mechanics, 108, 67-88.

[5] Mavrakos, S.A. \& McIver, P. 1997. Comparison of methods for computing hydrodynamic characteristics of arrays of wave power devices. Applied Ocean Research, 19, 283-291.

[6] Thomas, G. 2008. The theory behind the conversion of ocean wave energy: a review. In: Ocean Wave Energy (J. Cruz editor). Springer, Germany.

[7] Garnaud, X. \& Mei, C.C. 2009. Bragg scattering and wave-power extraction by an array of small buoys. Proceedings Royal Society London A, 466, 79-106.

[8] Babarit, A., 2010. Impact of long separating distances on the energy production of two interacting wave energy converters. Ocean Engineering, $37,718-729$.

[9] Falnes, J. \& Hals, J. 2012. Heaving buoys, point absorbers and arrays. Philosophic Transactions Royal Society A, 370, 246-277. 
[10] Babarit, A. 2013. On the park effect in arrays of oscillating wave energy converters. Renewable Energy, 58, 68-78.

[11] Whittaker, T. \& Folley ,M. 2012. Nearshore oscillating wave surge converters and the development of Oyster. Philosophic Transactions Royal Society A, 370, 345-364.

[12] Mei, C.C., Stiassnie, M.\& Yue, D. K.-P. 2005. Theory and applications of ocean surface waves. World Scientific, USA.

[13] Rafiee, A. \& Dias, F. 2012. Numerical simulation of wave impact on an oscillating wave surge converter. Proceedings of the 2nd International Conference on Violent Flows, Nantes (France), 157-163.

[14] Rafiee, A. \& Dias, F. 2013. Two-dimensional and three-dimensional simulation of wave interaction with an Oscillating Wave Surge Converter. International Workshop on Water Waves and Floating Bodies (IWWWFB) 2013, France.

[15] Rafiee, A., Elsaesser, B. \& Dias, F. 2013. Numerical Simulation of Wave Interaction with an Oscillating Wave Surge Converter. Proceedings of the 32nd International Conference on Ocean, Offshore and Arctic Engineering (OMAE 2013), Nantes.

[16] Wei, Y., Rafiee, A., Elsaesser, B. \& Dias, F. 2013. Numerical Simulation of an Oscillating Wave Surge Converter. Proceedings of the 32nd International Conference on Ocean, Offshore and Arctic Engineering (OMAE 2013), Nantes.

[17] Sarkar, D., Renzi, E., \& Dias, F., 2013. Wave power extraction by an oscillating wave surge converter in random sea. Proceedings of the 32nd International Conference on Ocean, Offshore and Arctic Engineering (OMAE 2013), Nantes.

[18] Henry, A., Doherty, K., Cameron, L., Whittaker, T. \& Doherty, R. 2010. Advances in the design of the Oyster wave energy converter, RINA Marine and Offshore Energy Conference, London, UK.

[19] Renzi, E. \& Dias, F. 2012. Resonant behaviour of an oscillating wave energy converter in a channel. Journal of Fluid Mechanics, 701, 482-510. 
[20] Renzi, E. \& Dias, F. 2013. Relations for a periodic array of flap-type wave energy converters. Applied Ocean Research, 39, 31-39.

[21] Babarit, A., Hals, J., Muliawan, M.J., Kurniawan, A., Moan, T., Krokstad, J. 2012. Numerical benchmarking study of a selection of wave energy converters. Renewable Energy, 41, 44-63.

[22] Renzi, E. \& Dias, F. 2013. Hydrodynamics of the Oscillating Wave Surge Converter in the open ocean. European Journal of Mechanics B/Fluids, DOI:10.1016/j.euromechflu.2013.01.007.

[23] Renzi, E. \& Dias, F. 2013. Mathematical modelling of a flap-type wave energy converter. Proceedings of the 32nd International Conference on Ocean, Offshore and Arctic Engineering (OMAE 2013), Nantes.

[24] Kagemoto, H. \& Yue, D.K.P. 1986. Interactions among multiple threedimensional bodies in water waves: an exact algebraic method. Journal of Fluid Mechanics, 166, 189-209.

[25] Mei, C.C., Sammarco, P., Chan E.S. \& Procaccini C. 1994. Subharmonic Resonance of Proposed Storm Gates for Venice Lagoon. Proceedings Royal Society London A, 444, 257-265.

[26] Folley, M., Babarit, A., Child, B., Forehand, D., O'Boyle, L., Silverthorne, K., Spinneken, J., Stratigaki, V., Troch, P., 2012. A review of numerical modelling of wave energy converters. Proceedings of the ASME 31st International Conference on Ocean, Offshore and Arctic Engineering (OMAE 2012), Rio de Janeiro, Brazil.

[27] Newman, J.N. 2001. Wave Effects on Multiple Bodies. In: Hydrodynamics in Ship and Ocean Engineering (M. Kashiwagi editor), RIAM, Kyushu University, Japan.

[28] McIver, P. 2002. Wave interaction with arrays of structures. Applied Ocean Research, 24, 121-126.

[29] Adamo, A. \& Mei, C.C., 2005. Linear response of Venice storm gates to incident waves. Proceedings Royal Society London A, 461, 1711-1734.

[30] Siddorn, P. \& Eatock Taylor, R. 2008. Diffraction and independent radiation by an array of floating cylinders. Ocean Engineering, 35, 12891303. 
[31] Sammarco P., Tran H.H. \& Mei C.C. 1997a. Subharmonic resonance of Venice gates in waves. Part 1. Evolution equation and uniform incident waves. Journal of Fluid Mechanics, 349, 295-325.

[32] Sammarco P., Tran H.H., Gottlieb O., Mei C.C. 1997b. Subharmonic resonance of Venice gates in waves. Part 2. Sinusoidally modulated incident waves. Journal of Fluid Mechanics, 349, 327-359.

[33] Whittaker, T., Collier, D., Folley, M., Osterried, M., Henry, A. and Crowley, M., 2007. The development of Oyster - A shallow water surging wave energy converter. Proceedings of the 7th European Wave and Tidal Energy Conference, Porto (Portugal).

[34] Wehausen, J.V. 1971. The motion of floating bodies. Annual Review of Fluid Mechanics, 3, 237-268.

[35] Kara, F. 2010. Time domain prediction of power absorption from ocean waves with latching control. Renewable Energy, 35 (2), 423-434.

[36] Givoli, D. 1991. Non-reecting boundary conditions. Journal of Computational Physics 94 (1), 1-29.

[37] Givoli, D. 1992. A numerical-solution procedure for exterior wave problems. Computers and Structures 43 (1), 77-84.

[38] Tam, P.T. 2008. A Physicist's Guide to Mathematica ${ }^{\circledR}$. Academic Press, USA.

[39] Sammarco, P. \& Renzi, E. 2007. Wave actions on the side caissons of the Venice gates. Applied Ocean Research, 29, 210-220.

[40] Maniar, H.D. \& Newman, J.N. 1997. Wave diffraction by a long array of cylinders. Journal of Fluid Mechanics, 339, 309-330.

[41] Aquamarine Power Limited, 2012. Lewis Wave Power 40MW Oyster Wave Array Non Technical Summary. www.aquamarinepower.com.

[42] Chatjigeorgiou, I.K. 2013. The hydrodynamics of arrays of truncated elliptical cylinders. European Journal of Mechanics B/Fluids, 37, 153164. 
[43] Gradshteyn, I.S. \& Ryzhik, I.M. 2007. Table of Integrals, Series and Products. Academic Press, USA.

[44] Chung, J. \& Hulbert, G.M. 1993. A time integration algorithm for structural dynamics with improved numerical dissipation: The generalized- $\alpha$ method. Journal of Applied Mechanics, 60, 371-375.

[45] Jansen, K.E., Whiting, C.H. \& Hulbert, G.M. 2000. A generalized- $\alpha$ method for integrating the filtered NavierStokes equations with a stabilized finite element method. Comput. Methods Appl. Mech. Engrg., vol. 190, pp. 305-319. 